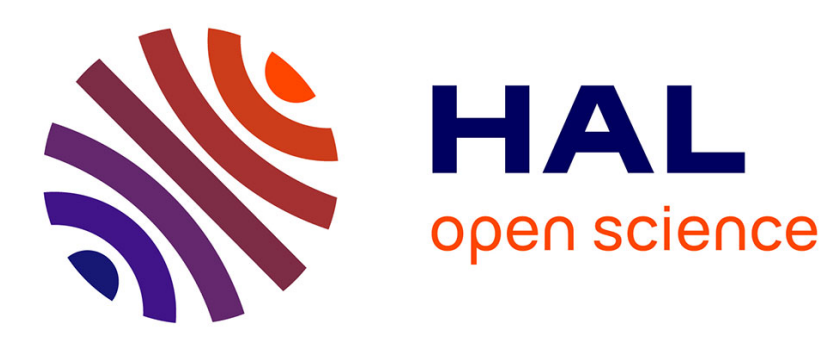

\title{
Two-phase inertial flow in homogeneous porous media: A theoretical derivation of a macroscopic model
}

\author{
Didier Lasseux, Azita Ahmadi, Ali Akbar Abbasian Arani
}

\section{To cite this version:}

Didier Lasseux, Azita Ahmadi, Ali Akbar Abbasian Arani. Two-phase inertial flow in homogeneous porous media: A theoretical derivation of a macroscopic model. Transport in Porous Media, 2008, 75 (3), pp.371-400. 10.1007/s11242-008-9231-y . hal-01174658

\section{HAL Id: hal-01174658 \\ https://hal.science/hal-01174658}

Submitted on 25 Oct 2017

HAL is a multi-disciplinary open access archive for the deposit and dissemination of scientific research documents, whether they are published or not. The documents may come from teaching and research institutions in France or abroad, or from public or private research centers.
L'archive ouverte pluridisciplinaire HAL, est destinée au dépôt et à la diffusion de documents scientifiques de niveau recherche, publiés ou non, émanant des établissements d'enseignement et de recherche français ou étrangers, des laboratoires publics ou privés. 


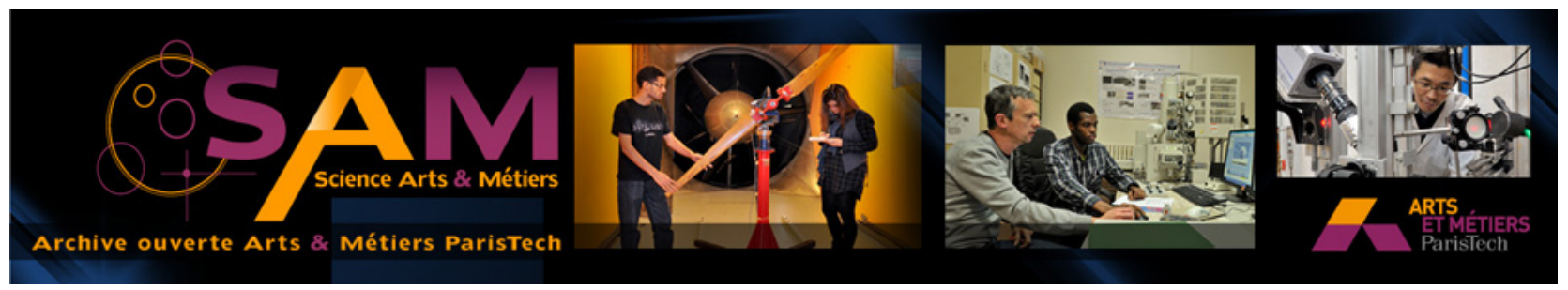

Science Arts \& Métiers (SAM)

is an open access repository that collects the work of Arts et Métiers ParisTech researchers and makes it freely available over the web where possible.

This is an author-deposited version published in: http://sam.ensam.eu

Handle ID: .http://hdl.handle.net/10985/9745

\section{To cite this version :}

Didier LASSEUX, Azita AHMADI, Ali Akbar ABBASIAN ARANI - Two-phase inertial flow in homogeneous porous media: A theoretical derivation of a macroscopic model - Transport in Porous Media - Vol. 75, n`3, p.371-400 - 2008 


\title{
Two-Phase Inertial Flow in Homogeneous Porous Media: A Theoretical Derivation of a Macroscopic Model
}

\author{
Didier Lasseux • Azita Ahmadi • \\ Ali Akbar Abbasian Arani
}

\begin{abstract}
The purpose of this article is to derive a macroscopic model for a certain class of inertial two-phase, incompressible, Newtonian fluid flow through homogenous porous media. Starting from the continuity and Navier-Stokes equations in each phase $\beta$ and $\gamma$, the method of volume averaging is employed subjected to constraints that are explicitly provided to obtain the macroscopic mass and momentum balance equations. These constraints are on the length- and time-scales, as well as, on some quantities involving capillary, Weber and Reynolds numbers that define the class of two-phase flow under consideration. The resulting macroscopic momentum equation relates the phase-averaged pressure gradient $\nabla\left\langle p_{\alpha}\right\rangle^{\alpha}$ to the filtration or Darcy velocity $\left\langle\mathbf{v}_{\alpha}\right\rangle$ in a coupled nonlinear form explicitly given by
\end{abstract}

$$
\begin{aligned}
\left\langle\mathbf{v}_{\alpha}\right\rangle= & -\frac{\mathbf{K}_{\alpha \alpha}^{*}}{\mu_{\alpha}} \cdot\left(\nabla\left\langle p_{\alpha}\right\rangle^{\alpha}-\rho_{\alpha} \mathbf{g}\right)-\mathbf{F}_{\alpha \alpha} \cdot\left\langle\mathbf{v}_{\alpha}\right\rangle \\
& -\frac{\mathbf{K}_{\alpha \kappa}^{*}}{\mu_{\kappa}} \cdot\left(\nabla\left\langle p_{\kappa}\right\rangle^{\kappa}-\rho_{\kappa} \mathbf{g}\right)-\mathbf{F}_{\alpha \kappa} \cdot\left\langle\mathbf{v}_{\kappa}\right\rangle \quad \alpha, \kappa=\beta, \gamma \quad \alpha \neq \kappa
\end{aligned}
$$

or equivalently

$$
\begin{aligned}
\left\langle\mathbf{v}_{\alpha}\right\rangle= & -\frac{\mathbf{K}_{\alpha}}{\mu_{\alpha}} \cdot\left(\nabla\left\langle p_{\alpha}\right\rangle^{\alpha}-\rho_{\alpha} \mathbf{g}\right)-\mathbf{F}_{\alpha \alpha} \cdot\left\langle\mathbf{v}_{\alpha}\right\rangle \\
& +\mathbf{K}_{\alpha \kappa} \cdot\left\langle\mathbf{v}_{\kappa}\right\rangle-\mathbf{F}_{\alpha \kappa} \cdot\left\langle\mathbf{v}_{\kappa}\right\rangle \quad \alpha, \kappa=\beta, \gamma \quad \alpha \neq \kappa
\end{aligned}
$$

In these equations, $\mathbf{F}_{\alpha \alpha}$ and $\mathbf{F}_{\alpha \kappa}$ are the inertial and coupling inertial correction tensors that are functions of flow-rates. The dominant and coupling permeability tensors $\mathbf{K}_{\alpha \alpha}^{*}$ and $\mathbf{K}_{\alpha \kappa}^{*}$ and the permeability and viscous drag tensors $\mathbf{K}_{\alpha}$ and $\mathbf{K}_{\alpha \kappa}$ are intrinsic and are those defined in the conventional manner as in (Whitaker, Chem Eng Sci 49:765-780, 1994) and (Lasseux et al., Transport Porous Media 24(1):107-137, 1996). All these tensors can be determined from closure problems that are to be solved using a spatially periodic model of a porous medium. The practical procedure to compute these tensors is provided.

D. Lasseux $(\varangle) \cdot$ A. Ahmadi · A. A. Abbasian Arani

TREFLE - UMR CNRS 8508 - University Bordeaux 1, Esplanade des Arts et Metiers,

Talence 33405, France

e-mail: didier.lasseux@bordeaux.ensam.fr 
Keywords Homogeneous porous media - Two-phase flow · Inertial or non-Darcian flow · Up-scaling · Volume averaging

\section{Notations}

$\mathbf{a}_{\alpha \kappa} \quad$ A vector that maps $\mu_{\alpha}\left\langle\mathbf{v}_{\kappa}\right\rangle^{\kappa}$ onto $\tilde{p}_{\alpha}, \mathrm{m}^{-1}$

$\mathbf{A}_{\alpha \kappa} \quad$ A tensor that maps $\left\langle\mathbf{v}_{\kappa}\right\rangle^{\kappa}$ onto $\tilde{\mathbf{v}}_{\alpha}$

$A_{\alpha e} \quad$ Area of $\alpha$-phase entrances and exits associated with the macroscopic region, $\mathrm{m}^{2}$

$A_{\alpha \kappa} \quad$ Area of $\alpha-\kappa$ interface contained within the averaging volume $\left(=A_{\kappa \alpha}\right), \mathrm{m}^{2}$

$C a_{\alpha} \quad$ Capillary number associated to the $\alpha$-phase $\left(=\frac{\mu_{\alpha}\left\|\left\langle\mathbf{v}_{\alpha}\right\rangle^{\alpha}\right\|}{\sigma}\right)$

$\mathbf{F}_{\alpha} \quad$ Inertial correction tensor for the $\alpha$-phase

$\mathbf{F}_{\alpha \kappa} \quad$ Coupling inertial correction tensor that maps $\left\langle\mathbf{v}_{\kappa}\right\rangle$ onto $\left\langle\mathbf{v}_{\alpha}\right\rangle$

g Gravitational acceleration, $\mathrm{m} \mathrm{s}^{-2}$

$H \quad$ Mean curvature, $\mathrm{m}^{-1}$

$\langle H\rangle_{\beta \gamma} \quad$ Area average over $A_{\beta \gamma}$ of the mean curvature, $\mathrm{m}^{-1}$

I Unit tensor

$\mathbf{K}_{\alpha} \quad$ Permeability tensor for the $\alpha$-phase, $\mathrm{m}^{2}$

$\mathbf{K}_{\alpha \kappa} \quad$ Viscous drag tensor that maps $\left\langle\mathbf{v}_{\kappa}\right\rangle$ onto $\left\langle\mathbf{v}_{\alpha}\right\rangle$

$\mathbf{K}_{\alpha \alpha}^{*} \quad$ Dominant permeability tensor that maps $\left.\left(\nabla\left\langle p_{\alpha}\right\rangle^{\alpha}-\rho_{\alpha} \mathbf{g}\right) / \mu_{\alpha}\right)$ onto $\left\langle\mathbf{v}_{\alpha}\right\rangle, \mathrm{m}^{2}$

$\mathbf{K}_{\alpha \kappa}^{*} \quad$ Dominant permeability tensor that maps $\left.\left(\nabla\left\langle p_{\kappa}\right\rangle^{\kappa}-\rho_{\kappa} \mathbf{g}\right) / \mu_{\kappa}\right)$ onto $\left\langle\mathbf{v}_{\alpha}\right\rangle, \mathrm{m}^{2}$

$l_{\alpha} \quad$ Characteristic length for the $\alpha$-phase, $\mathrm{m}$

$\mathbf{l}_{i} \quad i=1,2,3$, lattice vectors, $\mathrm{m}$

$l_{p} \quad$ Small length scale representation of the mean pore diameter, $\mathrm{m}$

$L \quad$ Characteristic length associated with volume averaged quantities, $m$

$\mathbf{n}_{\alpha \kappa} \quad$ Unit normal vector pointing from the $\alpha$-phase towards the $\kappa$-phase $\left(=-\mathbf{n}_{\kappa \alpha}\right)$

$\mathbf{n}_{\beta} \quad$ Unit normal vector representing both $\mathbf{n}_{\beta \gamma}$ and $\mathbf{n}_{\beta \sigma}$

$\mathbf{n}_{\gamma} \quad$ Unit normal vector representing both $\mathbf{n}_{\gamma \beta}$ and $\mathbf{n}_{\gamma \sigma}$

$p_{\alpha} \quad$ Pressure in the $\alpha$-phase, $P a$

$\left\langle p_{\alpha}\right\rangle \quad$ Superficial average pressure in the $\alpha$-phase, $\mathrm{Pa}$

$\left\langle p_{\alpha}\right\rangle^{\alpha} \quad$ Intrinsic average pressure in the $\alpha$-phase, $\mathrm{Pa}$

$\tilde{p}_{\alpha} \quad$ Pressure deviation in the $\alpha$-phase $\left(=p_{\alpha}-\left\langle p_{\alpha}\right\rangle^{\alpha}\right), \mathrm{Pa}$

$p_{\alpha}^{0} \quad$ Reference pressure in the $\alpha$-phase, $\mathrm{Pa}$

$r_{0} \quad$ Radius of the averaging volume $V, \mathrm{~m}$

r Position vector, $\mathrm{m}$

$R e_{\alpha} \quad$ Reynolds number associated to the $\alpha$-phase, $\left(=\frac{\rho_{\alpha}\left\|\left\langle\mathbf{v}_{\alpha}\right\rangle\right\| l_{p}}{\mu_{\alpha}}\right)$

$t \quad$ Time, $\mathrm{s}$

$t^{*} \quad$ Characteristic process time, $\mathrm{s}$

$\mathbf{v}_{\alpha} \quad$ Velocity in the $\alpha$-phase, $\mathrm{m} \mathrm{s}^{-1}$

$\left\langle\mathbf{v}_{\alpha}\right\rangle \quad$ Superficial average velocity in the $\alpha$-phase, $\mathrm{m} \mathrm{s}^{-1}$

$\left\langle\mathbf{v}_{\alpha}\right\rangle^{\alpha} \quad$ Intrinsic average velocity in the $\alpha$-phase, $\mathrm{m} \mathrm{s}^{-1}$

$\widetilde{\mathbf{v}}_{\alpha} \quad$ Velocity deviation in the $\alpha$-phase $\left(=\mathbf{v}_{\alpha}-\left\langle\mathbf{v}_{\alpha}\right\rangle^{\alpha}\right), \mathrm{m} \mathrm{s}^{-1}$

$V_{\alpha} \quad$ Volume of the $\alpha$-phase, contained within the averaging volume, $\mathrm{m}^{3}$

$V \quad$ Averaging volume, $\mathrm{m}^{3}$

$W e_{\alpha} \quad$ Weber number associated to the $\alpha$-phase $\left(=\frac{\rho_{\alpha}\left\|\left\langle\mathbf{v}_{\alpha}\right\rangle^{\alpha}\right\|^{2} l_{p}}{\sigma}\right)$

$\mathbf{y}_{\alpha} \quad$ Position of a point in the $\alpha$-phase relative to the centroid of $V, \mathrm{~m}$ 


\section{Greek letters}

$\delta_{\alpha \kappa} \quad$ Kronecker delta function ( $\delta_{\alpha \kappa}=1$ if $\alpha=\kappa, 0$ otherwise)

$\varepsilon_{\alpha} \quad$ Volume fraction of the $\alpha$-phase, $\left(=V_{\alpha} / V\right)$

$\mu_{\alpha} \quad$ Viscosity of the $\alpha$-phase, Pas

$\rho_{\alpha} \quad$ Density of the $\alpha$-phase, $\mathrm{kg} / \mathrm{m}^{3}$

$\sigma \quad$ Interfacial tension between the $\beta$ - and $\gamma$-phase $\mathrm{N} / \mathrm{m}$

$\mathbf{T}_{v \alpha} \quad$ Viscous stress tensor in the $\alpha$-phase, $\mathrm{Pa}$

\section{Introduction}

Fluid flow through porous media is of interest in several domains, such as petroleum recovery, chemical and environmental engineering. Although Darcy's law (Darcy 1856) and its generalized form for two-phase flow (Muskat 1937; Raats and Klute 1968) remain physically relevant for a wide range of applications, problems for which these models fail to correctly represent flow rate to pressure drop relationships are of considerable practical importance. Pertaining to this class of problems are flows occurring in packed beds of reactors and in many subsurface systems such as flow near wells of oil or gas production, water pumping and soil remediation to cite a few. Within this context, flow combining both inertial and multiphase conditions represent challenging issues that still deserve special attention.

While the initial empirical one- and two-phase versions of Darcy's law have been supported experimentally, numerically and theoretically (Whitaker 1986a,b; Auriault 1987), and while studies of one-phase inertial flow has also received significant efforts, the same strong support is still lacking for the inertial two-phase flow. In studies of non-Darcy flow through porous media, the Forchheimer equation involving a quadratic velocity correction is generally used to describe single phase inertial flow. Originally put forth on an empirical basis (Forchheimer 1901), this equation has been extensively employed to interpret experimental data (Ergun 1952; Ward 1964; Beavers and Sparrow 1969; Dullien and Azzam 1973; MacDonald et al. 1979). It was also used to analyze numerical results (Coulaud et al. 1988; Ma and Ruth 1993; Ruth and Ma 1993; Thauvin and Mohanty 1998; Papathanasiou et al. 2001). During the same period, this model found some theoretical justifications (Irmay 1958; Blick 1966; Ahmed and Sunada 1969; Cvetkovic 1986; Giorgi 1997; Chen et al. 2001). More refinements demonstrating the existence of different regimes (weak and strong inertia) were however reported from numerical simulation results obtained on very different structures (Firdaouss and Guermond 1995; Firdaouss et al. 1997; Koch and Ladd 1997; Amaral Souto and Moyne 1997; Rojas and Koplik 1998; Skjetne et al. 1999). The existence of the weak and strong inertia regimes was also identified from a theoretical point of view (Wodie and Levy 1991; Mei and Auriault 1991; Rasoloarijaona and Auriault 1994; Skjetne and Auriault 1999). A more general form of the inertial flow of a single $\beta$-phase in a homogeneous porous medium given by

$$
\left\langle\mathbf{v}_{\beta}\right\rangle=\frac{-\mathbf{K}}{\mu_{\beta}} \cdot\left[\nabla\left\langle p_{\beta}\right\rangle^{\beta}-\rho_{\beta} \mathbf{g}\right]-\mathbf{F} \cdot\left\langle\mathbf{v}_{\beta}\right\rangle
$$

was recently proposed (Whitaker 1996) where $\mathbf{K}$ and $\mathbf{F}$ are, respectively, the Darcy's law (intrinsic) permeability and non-Darcy (Forchheimer) velocity dependent correction tensors. These two tensors can be determined from the solution of periodic closure problems that are solved on a representative periodic unit cell of the porous medium.

Several studies reported in the literature extend the Forchheimer equation to multiphase flow on an empirical basis and provide relationships for correlating non-Darcy flow coefficients 
under multiphasic conditions (Evans et al. 1987; Evans and Evans 1988; Liu et al. 1995). Similar models were also considered in nuclear safety applications (Buchlin and Stubos 1987; Lipinski 1980, 1982). Some attempt was made to extend to porous media the LockhartMartinelli model initially derived for two-phase flow in pipes (Lockhart and Martinelli 1949; Fourar and Lenormand 2000). A theoretical derivation of a macroscopic model was proposed by Bennethum and Giorgi (1997) using the hybrid mixture theory, without any means, however, to estimate the macroscopic coefficients appearing in the macroscopic model for further comparison to experimental data. Nevertheless, the Forchheimer model and its generalized form, the degeneration of which at vanishing Reynolds number correctly restores Darcy's law, have now become some standard models to describe high-velocity flow in petroleum engineering (Bear 1972; Scheidegger 1974; Geertsma 1974; Firoozabadi and Katz 1979; Firoozabadi et al. 1995). In this context, thorough theoretical analysis still leaves much to be desired in order to fully understand high-rate or inertial flow through porous media and overcome mathematical difficulties in handling highly nonlinear multiphase flow equations outside the Darcy regime.

The objective of the present study is to analyze the possibility of deriving a macroscopic model describing inertial (but laminar) two-phase, incompressible, Newtonian fluid flow through homogenous porous media using the method of volume averaging. Starting from the two-phase Navier-Stokes boundary value problem and following lines developed in previous works (Whitaker 1986b, 1994; Lasseux et al. 1996; Whitaker 1996), the theoretical derivation of the averaged form of the mass and momentum balance equations is achieved under the restriction of constraints on the length- and time-scales as well as on dimensionless quantities involving the capillary, Weber and Reynolds numbers. These constraints are explicitly provided during the course of the development and specify the class of inertial two-phase flow under consideration.

\section{Microscopic Boundary Value Problem}

The process under consideration is the simultaneous flow of two immiscible phases $\beta$ and $\gamma$ both incompressible and Newtonian in a porous medium that is composed of a rigid inert solid phase $\sigma$. The macroscopic region with the characteristic length, $L$, illustrated in Fig. 1 represents a homogeneous porous medium with respect to the two-phase flow process.

The boundary-value problem describing the flow at the pore-scale in the macroscopic region is given by the following set of equations

$$
\begin{gathered}
\rho_{\alpha}\left(\frac{\partial \mathbf{v}_{\alpha}}{\partial t}+\mathbf{v}_{\alpha} \cdot \nabla \mathbf{v}_{\alpha}\right)=-\nabla p_{\alpha}+\rho_{\alpha} \mathbf{g}+\mu_{\alpha} \nabla^{2} \mathbf{v}_{\alpha} \quad \text { in the } \alpha \text {-phase, } \quad \alpha=\beta \text { and } \gamma \\
\nabla \cdot \mathbf{v}_{\alpha}=0 \quad \text { in the } \alpha \text {-phase, } \alpha=\beta \text { and } \gamma \\
\text { BC1 and BC2 } \quad \mathbf{v}_{\alpha}=0 \quad \text { at } A_{\alpha \sigma}, \quad \alpha=\beta \text { and } \gamma \\
\text { BC3 } \quad \mathbf{v}_{\beta}=\mathbf{v}_{\gamma} \quad \text { at } A_{\beta \gamma} \\
\text { BC4 - } \mathbf{n}_{\beta \gamma} p_{\beta}+\mathbf{n}_{\beta \gamma} \cdot \mathbf{T}_{v \beta}=-\mathbf{n}_{\beta \gamma} p_{\gamma}+\mathbf{n}_{\beta \gamma} \cdot \mathbf{T}_{v \gamma}+2 \sigma H \mathbf{n}_{\beta \gamma} \quad \text { at } A_{\beta \gamma} \\
\text { BC5 and BC6 } \quad \mathbf{v}_{\alpha}=\mathbf{f}_{\alpha}(t) \quad \text { at } A_{\alpha e}, \quad \alpha=\beta \text { and } \gamma
\end{gathered}
$$




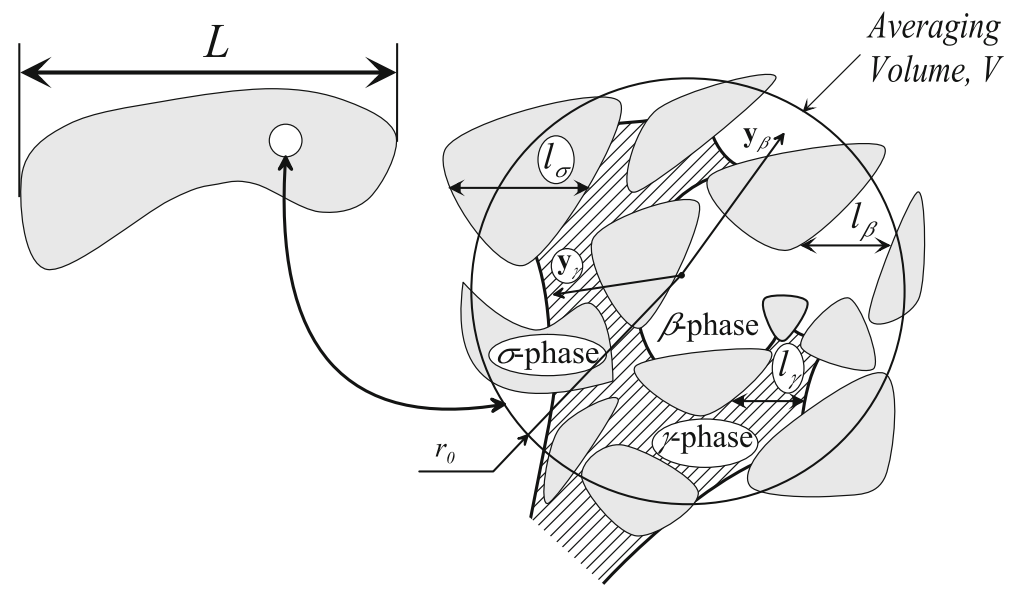

Fig. 1 Macroscopic region and averaging volume

In these equations $A_{\beta \gamma}$ and $A_{\alpha \sigma}$ represent the $\beta-\gamma$ and $\alpha-\sigma$ interfaces contained in the macroscopic region (see Fig. 1) while $A_{\alpha e}(\alpha=\beta, \gamma)$ represents the $\alpha$-phase entrances and exits of that region.

Equation 6, in which the interfacial tension is represented by $\sigma$ and the mean curvature of the interface by $H$, expresses the balance of the stress jump, which is purely normal, by capillary effects. In the presentation of this condition, we have used the assumption which ignores effects of surface-active agents that are always present in real two-phase systems, (Slattery 1990). The total $\alpha$-phase stress tensor, $\mathbf{T}_{\alpha}(\alpha=\beta, \gamma)$, has been decomposed according to

$$
\mathbf{T}_{\alpha}=-p_{\alpha} \mathbf{I}+\mathbf{T}_{v \alpha}
$$

and, in Eq. 2, according to the Newtonian character of the $\alpha$-phase, the viscous stress tensor $\mathbf{T}_{v \alpha}(\alpha=\beta, \gamma)$ is taken as

$$
\mathbf{T}_{v \alpha}=\mu_{\alpha}\left(\nabla \mathbf{v}_{\alpha}+\nabla \mathbf{v}_{\alpha}^{T}\right)
$$

The above system of equations is not complete until the level of the pressure field is specified, and this is done by specifying $p_{\alpha}$ at some point

$$
\text { BC7 } \quad p_{\alpha}=p_{\alpha}^{0} \quad \mathbf{r}=\mathbf{r}^{0} \quad \alpha=\beta \text { or } \gamma
$$

\section{Volume Averaging}

Although the method of weighted averages with appropriate weighting functions could appear as an appealing one (Marle 1967; Quintard and Whitaker 1994a), we chose to use the volume averaging technique for the sake of simplicity and to avoid adding excessive complexity in the developments and notations. This choice is further motivated by the fact that our development can be significantly shortened since the average of the Stokes part of the momentum equation (2) and the closure problems used to determine permeability tensors have already been presented in previous works using this technique (Whitaker 1986b; Lasseux et al. 1996; Whitaker 1994). When this technique is employed, special attention must however be dedicated to the definition of the averaging volume, in particular for ordered structures, and to the 
order of magnitude of terms associated to phase spatial moments (see Quintard and Whitaker 1994c).

Averaging of the above boundary value problem is performed over a volume $V$ represented in Fig. 1 including the volumes $V_{\alpha}(\alpha=\beta, \gamma)$ of the two phases of respective volume fractions $\varepsilon_{\alpha}(\alpha=\beta, \gamma)$ given by

$$
\varepsilon_{\alpha}=\frac{V_{\alpha}}{V}
$$

In the course of the averaging process, we shall use the superficial and intrinsic averages which, for any quantity $\psi_{\alpha}$ defined in $V_{\alpha}$, are, respectively, given by

$$
\left\langle\psi_{\alpha}\right\rangle=\frac{1}{V} \int_{V_{\alpha}} \psi_{\alpha} d V \quad \alpha=\beta, \gamma
$$

and

$$
\left\langle\psi_{\alpha}\right\rangle^{\alpha}=\frac{1}{V_{\alpha}} \int_{V_{\alpha}} \psi_{\alpha} d V \quad \alpha=\beta, \gamma
$$

with the evident relationship

$$
\left\langle\psi_{\alpha}\right\rangle=\varepsilon_{\alpha}\left\langle\psi_{\alpha}\right\rangle^{\alpha} \quad \alpha=\beta, \gamma
$$

Derivation of the averaged form of the mass and momentum equations requires the use of the averaging theorem for a three-phase system (Howes and Whitaker 1985). For some scalar quantity $\psi_{\alpha}$ associated with the $\alpha$-phase, this theorem takes the form

$$
\left\langle\nabla \psi_{\alpha}\right\rangle=\nabla\left\langle\psi_{\alpha}\right\rangle+\frac{1}{V} \int_{A_{\alpha \sigma}} \mathbf{n}_{\alpha} \psi_{\alpha} d A+\frac{1}{V} \int_{A_{\beta \gamma}} \mathbf{n}_{\alpha} \psi_{\alpha} d A \quad \alpha=\beta, \gamma
$$

In this relationship $A_{\alpha \sigma}$ and $A_{\beta \gamma}$, respectively, represent the interfacial areas between the $\alpha$ and $\sigma$ and $\beta$ and $\gamma$ phases contained within the averaging volume $V$ while $\mathbf{n}_{\alpha}$ is the unit normal vector pointing out of the $\alpha$-phase towards any of the other phases, solid or fluid. It must be noted that in Eq. 15 and throughout the entire article, we use, for compactness, the same symbol $\nabla$ to represent derivation with respect either to a macroscopic or to a microscopic space variable. Throughout the article and as in all up-scaling procedures, a constraint on scale hierarchy is assumed, namely

$$
l_{\alpha} \ll r_{0} \ll L \quad \alpha=\beta, \gamma
$$

In this relation, $l_{\alpha}$ represents the characteristic length within the $\alpha$-phase at the pore-scale and $r_{0}$ is the radius of the averaging volume, $V$.

\subsection{Continuity Equation}

The volume averaging procedure applied to the mass balance Eq. 3 is identical to the development of Whitaker (1986b) and leads to the macroscopic continuity equation given by

$$
\frac{\partial \varepsilon_{\alpha}}{\partial t}+\nabla \cdot\left\langle\mathbf{v}_{\alpha}\right\rangle=0 \quad \alpha=\beta, \gamma
$$

This equation is closed and does not require any special link with the microscopic scale. As will be seen below, this is not the case with the momentum balance equations. 
The next step is the volume averaging of the momentum balance equations and we begin with the average of the left-hand side (L.H.S.) of Eq. 2. Making use of the vector form of the transport theorem leads to (see Appendix A)

$$
\langle\text { L.H.S }\rangle=\rho_{\alpha}\left(\frac{\partial\left\langle\mathbf{v}_{\alpha}\right\rangle}{\partial t}-\frac{1}{V} \int_{A_{\beta \gamma}} \mathbf{n}_{\alpha} \cdot \mathbf{v}_{\alpha} \mathbf{v}_{\alpha} d A\right)+\rho_{\alpha}\left\langle\nabla \cdot\left(\mathbf{v}_{\alpha} \mathbf{v}_{\alpha}\right)\right\rangle \quad \alpha=\beta, \gamma
$$

The averaging theorem can be used in order to express the convective inertial term as

$$
\rho_{\alpha}\left\langle\nabla \cdot\left(\mathbf{v}_{\alpha} \mathbf{v}_{\alpha}\right)\right\rangle=\rho_{\alpha} \nabla \cdot\left\langle\mathbf{v}_{\alpha} \mathbf{v}_{\alpha}\right\rangle+\frac{\rho_{\alpha}}{V} \int_{A_{\beta \gamma}} \mathbf{n}_{\alpha} \cdot \mathbf{v}_{\alpha} \mathbf{v}_{\alpha} d A \quad \alpha=\beta, \gamma
$$

in which the no slip boundary condition in Eq. 4 was employed. At this stage, it is necessary to eliminate the average of the velocity product and to do so, we make use of the velocity decomposition given by Gray (1975)

$$
\mathbf{v}_{\alpha}=\left\langle\mathbf{v}_{\alpha}\right\rangle^{\alpha}+\tilde{\mathbf{v}}_{\alpha} \quad \alpha=\beta, \gamma
$$

When the intrinsic average of such a decomposition is considered and when $\left\langle\mathbf{v}_{\alpha}\right\rangle^{\alpha}$ experiences negligible variations within the averaging volume (at the scale $r_{0}$ ), we have

$$
\left\langle\left\langle\mathbf{v}_{\alpha}\right\rangle^{\alpha}\right\rangle^{\alpha}=\left\langle\mathbf{v}_{\alpha}\right\rangle^{\alpha} \quad \alpha=\beta, \gamma
$$

and accordingly

$$
\left\langle\tilde{\mathbf{v}}_{\alpha}\right\rangle^{\alpha}=0 \quad \alpha=\beta, \gamma
$$

For this to hold, it is necessary that terms like $\left\langle\mathbf{y}_{\alpha}\right\rangle \cdot \nabla\left\langle\mathbf{v}_{\alpha}\right\rangle^{\alpha},\left\langle\mathbf{y}_{\alpha} \mathbf{y}_{\alpha}\right\rangle: \nabla \nabla\left\langle\mathbf{v}_{\alpha}\right\rangle^{\alpha}$ (see Fig. 1) arising from Taylor developments are negligible and the requirement for this is essentially expressed by the scale hierarchy in Eq. 16 (Carbonell and Whitaker 1984; Whitaker 1999; Quintard and Whitaker 1994c). A more difficult problem might arise if phases are distributed on a regular periodic pattern. In this situation, however, a careful analysis of spatial moments performed on ordered and disordered structures suggests that a large enough convenient averaging volume (different from unit cells of the regular pattern) can be used (see Quintard and Whitaker 1994c). An alternative would be to define weighted averages (Quintard and Whitaker 1994b) at the cost, however, of a proper definition of the weighting functions which is beyond the scope of this work. Using the two Eqs. 21 and 22 allows to write

$$
\left\langle\mathbf{v}_{\alpha} \mathbf{v}_{\alpha}\right\rangle=\varepsilon_{\alpha}\left\langle\mathbf{v}_{\alpha}\right\rangle^{\alpha}\left\langle\mathbf{v}_{\alpha}\right\rangle^{\alpha}+\left\langle\tilde{\mathbf{v}}_{\alpha} \tilde{\mathbf{v}}_{\alpha}\right\rangle \quad \alpha=\beta, \gamma
$$

and hence

$$
\begin{aligned}
& \rho_{\alpha}\left\langle\nabla \cdot\left(\mathbf{v}_{\alpha} \mathbf{v}_{\alpha}\right)\right\rangle \\
& =\rho_{\alpha}\left(\nabla \cdot\left(\varepsilon_{\alpha}\left\langle\mathbf{v}_{\alpha}\right\rangle^{\alpha}\left\langle\mathbf{v}_{\alpha}\right\rangle^{\alpha}\right)+\nabla \cdot\left\langle\tilde{\mathbf{v}}_{\alpha} \tilde{\mathbf{v}}_{\alpha}\right\rangle+\frac{1}{V} \int_{A_{\beta \gamma}} \mathbf{n}_{\alpha} \cdot \mathbf{v}_{\alpha} \mathbf{v}_{\alpha} d A\right) \quad \alpha=\beta, \gamma
\end{aligned}
$$

When this result is inserted in Eq. 18 and when $\left\langle\mathbf{v}_{\alpha}\right\rangle^{\alpha}$ is employed instead of $\left\langle\mathbf{v}_{\alpha}\right\rangle$ we obtain

$$
\begin{aligned}
\langle\text { L.H.S }\rangle= & \rho_{\alpha} \varepsilon_{\alpha} \frac{\partial\left\langle\mathbf{v}_{\alpha}\right\rangle^{\alpha}}{\partial t}+\rho_{\alpha} \frac{\partial \varepsilon_{\alpha}}{\partial t}\left\langle\mathbf{v}_{\alpha}\right\rangle^{\alpha}+\rho_{\alpha} \nabla \cdot\left(\varepsilon_{\alpha}\left\langle\mathbf{v}_{\alpha}\right\rangle^{\alpha}\right)\left\langle\mathbf{v}_{\alpha}\right\rangle^{\alpha} \\
& +\rho_{\alpha} \varepsilon_{\alpha}\left\langle\mathbf{v}_{\alpha}\right\rangle^{\alpha} \cdot \nabla\left\langle\mathbf{v}_{\alpha}\right\rangle^{\alpha}+\rho_{\alpha} \nabla \cdot\left\langle\tilde{\mathbf{v}}_{\alpha} \tilde{\mathbf{v}}_{\alpha}\right\rangle \quad \alpha=\beta, \gamma
\end{aligned}
$$


Making use of the average mass balance equation allows to write the above equation as

$$
\langle\text { L.H.S }\rangle=\rho_{\alpha} \varepsilon_{\alpha} \frac{\partial\left\langle\mathbf{v}_{\alpha}\right\rangle^{\alpha}}{\partial t}+\rho_{\alpha} \varepsilon_{\alpha}\left\langle\mathbf{v}_{\alpha}\right\rangle^{\alpha} \cdot \nabla\left\langle\mathbf{v}_{\alpha}\right\rangle^{\alpha}+\rho_{\alpha} \nabla \cdot\left\langle\tilde{\mathbf{v}}_{\alpha} \tilde{\mathbf{v}}_{\alpha}\right\rangle \quad \alpha=\beta, \gamma
$$

At this point, we focus our interest on the right-hand side (R.H.S.) of Eq. 2. Since, this part of the momentum balance equation remains unchanged compared to the Stokes problem and the corresponding development of the generalized Darcy's law has been extensively presented in other works (Whitaker 1986b, 1994; Lasseux et al. 1996), we only provide the averaged form of this expression. Making use of the hierarchy of scales (Eq. 16) and the spatial decomposition of the velocity given in Eq. 20 and of the pressure given by

$$
p_{\alpha}=\left\langle p_{\alpha}\right\rangle^{\alpha}+\tilde{p}_{\alpha} \quad \alpha=\beta, \gamma
$$

the average of the (R.H.S.) of Eq. 2 takes the following form

$$
\begin{aligned}
\langle\text { R.H.S }\rangle= & -\nabla\left\langle p_{\alpha}\right\rangle+\varepsilon_{\alpha} \rho_{\alpha} \mathbf{g}+\mu_{\alpha} \nabla^{2}\left\langle\mathbf{v}_{\alpha}\right\rangle+\frac{1}{V} \int_{A_{\alpha \sigma} \cup A_{\beta \gamma}} \mathbf{n}_{\alpha} \cdot\left(-\tilde{p}_{\alpha} \mathbf{I}+\mu_{\alpha} \nabla \tilde{\mathbf{v}}_{\alpha}\right) d A \\
& -\nabla \varepsilon_{\alpha} \cdot\left(-\left\langle p_{\alpha}\right\rangle^{\alpha} \mathbf{I}+\mu_{\alpha} \nabla\left\langle\mathbf{v}_{\alpha}\right\rangle^{\alpha}\right) \quad \alpha=\beta, \gamma
\end{aligned}
$$

Reassembling Eqs. 26 and 28, replacing the superficial average pressure by its corresponding intrinsic form and dividing the result by $\varepsilon_{\alpha}$ allows us to express the average momentum balance equation in the $\alpha$-phase under the form

$$
\begin{aligned}
& \rho_{\alpha} \frac{\partial\left\langle\mathbf{v}_{\alpha}\right\rangle^{\alpha}}{\partial t}+\rho_{\alpha}\left\langle\mathbf{v}_{\alpha}\right\rangle^{\alpha} \cdot \nabla\left\langle\mathbf{v}_{\alpha}\right\rangle^{\alpha}+\rho_{\alpha} \varepsilon_{\alpha}^{-1} \nabla \cdot\left\langle\tilde{\mathbf{v}}_{\alpha} \tilde{\mathbf{v}}_{\alpha}\right\rangle \\
& =-\nabla\left\langle p_{\alpha}\right\rangle^{\alpha}+\rho_{\alpha} \underbrace{\mathbf{g}+\varepsilon_{\alpha}^{-1} \mu_{\alpha} \nabla^{2}\left\langle\mathbf{v}_{\alpha}\right\rangle-\mu_{\alpha} \varepsilon_{\alpha}^{-1} \nabla \varepsilon_{\alpha} \cdot \nabla\left\langle\mathbf{v}_{\alpha}\right\rangle^{\alpha}}_{\text {Brinkman correction }} \\
& \quad+\frac{1}{V_{\alpha}} \int_{A_{\alpha \sigma} \cup A_{\beta \gamma}} \mathbf{n}_{\alpha} \cdot\left(-\mathbf{I} \tilde{p}_{\alpha}+\mu_{\alpha} \nabla \tilde{\mathbf{v}}_{\alpha}\right) \mathrm{d} A \quad \alpha=\beta, \gamma
\end{aligned}
$$

In this last form, the three terms in the left-hand side correspond to the macroscopic acceleration and convective inertia while we can identify the two terms involving gradients of the average velocity in the right-hand side as the Brinkman correction terms. A simple order of magnitude analysis will indicate that these two terms are negligible. In fact, the order of magnitude of these Brinkman terms can be estimated to be

$$
\begin{gathered}
\varepsilon_{\alpha}^{-1} \mu_{\alpha} \nabla^{2}\left\langle\mathbf{v}_{\alpha}\right\rangle=\mathbf{O}\left(\frac{\mu_{\alpha}\left\langle\mathbf{v}_{\alpha}\right\rangle^{\alpha}}{L^{2}}\right) \quad \alpha=\beta, \gamma \\
\mu_{\alpha} \varepsilon_{\alpha}^{-1} \nabla \varepsilon_{\alpha} \cdot \nabla\left\langle\mathbf{v}_{\alpha}\right\rangle^{\alpha}=\mathbf{O}\left(\frac{\mu_{\alpha}\left\langle\mathbf{v}_{\alpha}\right\rangle^{\alpha}}{L^{2}}\right) \quad \alpha=\beta, \gamma
\end{gathered}
$$

In the latter of these two estimates, we have used the fact that $\varepsilon_{\beta}$ does not exhibit significant variations on length-scales shorter than $L$. Turning our attention to the interfacial viscous stress term appearing in the right-hand side of Eq. 29 indicates that

$$
\frac{\mu_{\alpha}}{V_{\alpha}} \int_{A_{\alpha \sigma} \cup A_{\beta \gamma}} \mathbf{n}_{\alpha} \cdot \nabla \tilde{\mathbf{v}}_{\alpha} \mathrm{d} A=\mathbf{O}\left(\frac{\mu_{\alpha}\left\langle\mathbf{v}_{\alpha}\right\rangle^{\alpha}}{l_{\alpha}} a_{\alpha}\right) \quad \alpha=\beta, \gamma
$$


Due to the no slip boundary condition at the fluid-solid interface $A_{\alpha \sigma}$ in conjunction with the decomposition in Eq. 20, we have used

$$
\tilde{\mathbf{v}}_{\alpha}=\mathbf{O}\left(\left\langle\mathbf{v}_{\alpha}\right\rangle^{\alpha}\right) \quad \alpha=\beta, \gamma
$$

and in Eq. 32 we have used $a_{\alpha}$ to designate the interfacial area per unit volume $\frac{A_{\alpha \sigma} \cup A_{\beta \gamma}}{V_{\alpha}}$. Providing a precise estimation of this last quantity is a difficult task. However, a lower bound of $a_{\alpha}$, given by $\frac{A_{\beta \gamma}}{V_{\alpha}}$, can be reasonably estimated to be on the order of $1 / l_{\alpha}$ leading to

$$
\frac{\mu_{\alpha}}{V_{\alpha}} \int_{A_{\alpha \sigma} \cup A_{\beta \gamma}} \mathbf{n}_{\alpha} \cdot \nabla \tilde{\mathbf{v}}_{\alpha} d A=\mathbf{O}\left(\frac{\mu_{\alpha}\left\langle\mathbf{v}_{\alpha}\right\rangle^{\alpha}}{l_{\alpha}^{2}}\right) \alpha=\beta, \gamma
$$

i.e.

$$
\varepsilon_{\alpha}^{-1} \mu_{\alpha} \nabla^{2}\left\langle\mathbf{v}_{\alpha}\right\rangle \ll \frac{\mu_{\alpha}}{V_{\alpha}} \int_{A_{\alpha \sigma} \cup A_{\beta \gamma}} \mathbf{n}_{\alpha} \cdot \nabla \tilde{\mathbf{v}}_{\alpha} d A \quad \alpha=\beta, \gamma
$$

and

$$
\mu_{\alpha} \varepsilon_{\alpha}^{-1} \nabla \varepsilon_{\alpha} \cdot \nabla\left\langle\mathbf{v}_{\alpha}\right\rangle^{\alpha} \ll \frac{\mu_{\alpha}}{V_{\alpha}} \int_{A_{\alpha \sigma} \cup A_{\beta \gamma}} \mathbf{n}_{\alpha} \cdot \nabla \tilde{\mathbf{v}}_{\alpha} \mathrm{d} A \quad \alpha=\beta, \gamma
$$

This indicates that Brinkman correction terms are unimportant in the macroscopic momentum equation and can be disregarded in the averaged form that can be written as

$$
\begin{aligned}
& \rho_{\alpha} \frac{\partial\left\langle\mathbf{v}_{\alpha}\right\rangle^{\alpha}}{\partial t}+\rho_{\alpha}\left\langle\mathbf{v}_{\alpha}\right\rangle^{\alpha} \cdot \nabla\left\langle\mathbf{v}_{\alpha}\right\rangle^{\alpha}+\rho_{\alpha} \varepsilon_{\alpha}^{-1} \nabla \cdot\left\langle\tilde{\mathbf{v}}_{\alpha} \tilde{\mathbf{v}}_{\alpha}\right\rangle=-\nabla\left\langle p_{\alpha}\right\rangle^{\alpha}+\rho_{\alpha} \mathbf{g} \\
& +\frac{1}{V_{\alpha}} \int_{A_{\alpha \sigma} \cup A_{\beta \gamma}} \mathbf{n}_{\alpha} \cdot\left(-\mathbf{I} \tilde{p}_{\alpha}+\mu_{\alpha} \nabla \tilde{\mathbf{v}}_{\alpha}\right) \mathrm{d} A \quad \alpha=\beta, \gamma
\end{aligned}
$$

At this stage of the development, the averaged momentum equations does not represent a closed form since velocity and pressure deviations having microscopic length-scale of variation are still present. Using this averaged form, we now need to derive the closure problem and this is detailed in the following section.

\section{Closure}

The objective of this section is to develop the relationships between spatial deviations and average quantities in order to close the macroscopic model.

\subsection{Continuity Equation}

Again, the development leading to the closure equation for mass balance has been presented in the literature (Whitaker 1986b, 1994; Lasseux et al. 1996) and we simply list the result as

$$
\nabla \cdot \tilde{\mathbf{v}}_{\alpha}=0 \quad \alpha=\beta, \gamma
$$

This result is subject to two constraints, the first one being the length-scale constraint expressed by Eq. 16 along with the order of magnitude given by Eq. 33 while the second one is a time-scale constraint that can be expressed as 


$$
t^{*} \gg \max _{\alpha=\beta, \gamma}\left(\frac{l_{\alpha}}{\left\|\left\langle\mathbf{v}_{\alpha}\right\rangle^{\alpha}\right\|}\right)
$$

This last relationship indicates that the flow process must be observed at times $t^{*}$ much larger than the characteristic time-scale of phase displacement at the pore-scale.

\subsection{Momentum Equation}

In order to develop the closure momentum equation, the averaged form in Eq. 37 is subtracted from the point momentum Eq. 2 providing the spatial deviations momentum equation that takes the form

$$
\begin{aligned}
& \rho_{\alpha} \frac{\partial \tilde{\mathbf{v}}_{\alpha}}{\partial t}+\rho_{\alpha} \mathbf{v}_{\alpha} \cdot \nabla \mathbf{v}_{\alpha}-\rho_{\alpha}\left\langle\mathbf{v}_{\alpha}\right\rangle^{\alpha} \cdot \nabla\left\langle\mathbf{v}_{\alpha}\right\rangle^{\alpha}-\rho_{\alpha} \varepsilon_{\alpha}^{-1} \nabla \cdot\left\langle\tilde{\mathbf{v}}_{\alpha} \tilde{\mathbf{v}}_{\alpha}\right\rangle=-\nabla \tilde{p}_{\alpha}+\mu_{\alpha} \nabla^{2} \tilde{\mathbf{v}}_{\alpha} \\
& +\mu_{\alpha} \nabla^{2}\left\langle\mathbf{v}_{\alpha}\right\rangle^{\alpha}-\frac{1}{V_{\alpha}} \int_{A_{\alpha \sigma} \cup A_{\beta \gamma}} \mathbf{n}_{\alpha} \cdot\left(-\mathbf{I} \tilde{p}_{\alpha}+\mu_{\alpha} \nabla \tilde{\mathbf{v}}_{\alpha}\right) \mathrm{d} A \quad \alpha=\beta, \gamma
\end{aligned}
$$

Given the orders of magnitude

$$
\mu_{\alpha} \nabla^{2} \tilde{\mathbf{v}}_{\alpha}=\mathbf{O}\left(\frac{\mu_{\alpha}\left\langle\mathbf{v}_{\alpha}\right\rangle^{\alpha}}{l_{\alpha}^{2}}\right) \quad \alpha=\beta, \gamma
$$

and

$$
\mu_{\alpha} \nabla^{2}\left\langle\mathbf{v}_{\alpha}\right\rangle^{\alpha}=\mathbf{O}\left(\frac{\mu_{\alpha}\left\langle\mathbf{v}_{\alpha}\right\rangle^{\alpha}}{L^{2}}\right) \quad \alpha=\beta, \gamma
$$

it is clear that this latter term can be discarded from Eq. 40. Moreover, by introducing the velocity spatial decomposition, the microscopic and the first of the two macroscopic inertial convective terms can be rearranged in the following way

$$
\begin{aligned}
& \rho_{\alpha} \mathbf{v}_{\alpha} \cdot \nabla \mathbf{v}_{\alpha}-\rho_{\alpha}\left\langle\mathbf{v}_{\alpha}\right\rangle^{\alpha} \cdot \nabla\left\langle\mathbf{v}_{\alpha}\right\rangle^{\alpha} \\
& =\rho_{\alpha}\left(\mathbf{v}_{\alpha} \cdot \nabla \tilde{\mathbf{v}}_{\alpha}+\mathbf{v}_{\alpha} \cdot \nabla\left\langle\mathbf{v}_{\alpha}\right\rangle^{\alpha}-\left\langle\mathbf{v}_{\alpha}\right\rangle^{\alpha} \cdot \nabla\left\langle\mathbf{v}_{\alpha}\right\rangle^{\alpha}\right) \\
& =\rho_{\alpha}\left(\mathbf{v}_{\alpha} \cdot \nabla \tilde{\mathbf{v}}_{\alpha}+\tilde{\mathbf{v}}_{\alpha} \cdot \nabla\left\langle\mathbf{v}_{\alpha}\right\rangle^{\alpha}\right) \quad \alpha=\beta, \gamma
\end{aligned}
$$

leading to the following momentum equation

$$
\begin{aligned}
& \rho_{\alpha} \frac{\partial \tilde{\mathbf{v}}_{\alpha}}{\partial t}+\rho_{\alpha} \mathbf{v}_{\alpha} \cdot \nabla \tilde{\mathbf{v}}_{\alpha}+\rho_{\alpha} \tilde{\mathbf{v}}_{\alpha} \cdot \nabla\left\langle\mathbf{v}_{\alpha}\right\rangle^{\alpha}-\rho_{\alpha} \varepsilon_{\alpha}^{-1} \nabla \cdot\left\langle\tilde{\mathbf{v}}_{\alpha} \tilde{\mathbf{v}}_{\alpha}\right\rangle \\
& =-\nabla \tilde{p}_{\alpha}+\mu_{\alpha} \nabla^{2} \tilde{\mathbf{v}}_{\alpha}-\frac{1}{V_{\alpha}} \int_{A_{\alpha \sigma} \cup A_{\beta \gamma}} \mathbf{n}_{\alpha} \cdot\left(-\mathbf{I} \tilde{p}_{\alpha}+\mu_{\alpha} \nabla \tilde{\mathbf{v}}_{\alpha}\right) d A \quad \alpha=\beta, \gamma
\end{aligned}
$$

This form can be significantly simplified when the following order of magnitude estimates, based on Eq. 33, are taken into account

$$
\begin{array}{r}
\mathbf{v}_{\alpha} \cdot \nabla \tilde{\mathbf{v}}_{\alpha}=\mathbf{O}\left(\frac{\left\|\left\langle\mathbf{v}_{\alpha}\right\rangle^{\alpha}\right\|^{2}}{l_{\alpha}}\right) \quad \alpha=\beta, \gamma \\
\tilde{\mathbf{v}}_{\alpha} \cdot \nabla\left\langle\mathbf{v}_{\alpha}\right\rangle^{\alpha}=\mathbf{O}\left(\frac{\left\|\left\langle\mathbf{v}_{\alpha}\right\rangle^{\alpha}\right\|^{2}}{L}\right) \quad \alpha=\beta, \gamma \\
\varepsilon_{\alpha}^{-1} \nabla \cdot\left\langle\tilde{\mathbf{v}}_{\alpha} \tilde{\mathbf{v}}_{\alpha}\right\rangle=\mathbf{O}\left(\frac{\left\|\left\langle\mathbf{v}_{\alpha}\right\rangle^{\alpha}\right\|^{2}}{L}\right) \quad \alpha=\beta, \gamma
\end{array}
$$


Here, we have used the fact that $\mathbf{v}_{\alpha}$ and $\tilde{\mathbf{v}}_{\alpha}$ are of the same order of magnitude, namely $\mathbf{O}\left(\left\langle\mathbf{v}_{\alpha}\right\rangle^{\alpha}\right)$, and this can be immediately inferred from Eqs. 20 and 33. On the basis of the length-scale constraint, this yields

$$
\begin{aligned}
& \rho_{\alpha} \mathbf{v}_{\alpha} \cdot \nabla \tilde{\mathbf{v}}_{\alpha} \gg \rho_{\alpha} \tilde{\mathbf{v}}_{\alpha} \cdot \nabla\left\langle\mathbf{v}_{\alpha}\right\rangle^{\alpha} \quad \alpha=\beta, \gamma \\
& \rho_{\alpha} \mathbf{v}_{\alpha} \cdot \nabla \tilde{\mathbf{v}}_{\alpha} \gg \rho_{\alpha} \varepsilon_{\alpha}^{-1} \nabla \cdot\left\langle\tilde{\mathbf{v}}_{\alpha} \tilde{\mathbf{v}}_{\alpha}\right\rangle \quad \alpha=\beta, \gamma
\end{aligned}
$$

As a consequence, the spatial deviations momentum equation takes the form

$$
\begin{aligned}
\rho_{\alpha} \frac{\partial \tilde{\mathbf{v}}_{\alpha}}{\partial t}+\rho_{\alpha} \mathbf{v}_{\alpha} \cdot \nabla \tilde{\mathbf{v}}_{\alpha}= & -\nabla \tilde{p}_{\alpha}+\mu_{\alpha} \nabla^{2} \tilde{\mathbf{v}}_{\alpha} \\
& -\frac{1}{V_{\alpha}} \int_{A_{\alpha \sigma} \cup A_{\beta \gamma}} \mathbf{n}_{\alpha} \cdot\left(-\mathbf{I} \tilde{p}_{\alpha}+\mu_{\alpha} \nabla \tilde{\mathbf{v}}_{\alpha}\right) \mathrm{d} A \quad \alpha=\beta, \gamma
\end{aligned}
$$

At this point, the closure problem remains non-steady and our interest is to provide a constraint for the explicit time dependent term to be negligible. This constraint can be easily formulated if we require the flow process to be observed at times $t^{*}$ much larger than the characteristic time of viscous relaxation at the pore scale, ${ }^{1}$ i.e.

$$
t^{*} \gg \max _{\alpha=\beta, \gamma}\left(\frac{\rho_{\alpha} l_{\alpha}^{2}}{\mu_{\alpha}}\right)
$$

Under these circumstances, since

$$
\rho_{\alpha} \frac{\partial \tilde{\mathbf{v}}_{\alpha}}{\partial t}=\mathbf{O}\left(\frac{\rho_{\alpha}\left\langle\mathbf{v}_{\alpha}\right\rangle^{\alpha}}{t^{*}}\right) \quad \alpha=\beta, \gamma
$$

it can be easily seen from Eq. 41 that

$$
\rho_{\alpha} \frac{\partial \tilde{\mathbf{v}}_{\alpha}}{\partial t} \ll \mu_{\alpha} \nabla^{2} \tilde{\mathbf{v}}_{\alpha} \quad \alpha=\beta, \gamma
$$

This leads to a closure equation for the momentum that takes the form

$$
\begin{aligned}
\rho_{\alpha} \mathbf{v}_{\alpha} \cdot \nabla \tilde{\mathbf{v}}_{\alpha}= & -\nabla \tilde{p}_{\alpha}+\mu_{\alpha} \nabla^{2} \tilde{\mathbf{v}}_{\alpha} \\
& -\frac{1}{V_{\alpha}} \int_{A_{\alpha \sigma} \cup A_{\beta \gamma}} \mathbf{n}_{\alpha} \cdot\left(-\mathbf{I} \tilde{p}_{\alpha}+\mu_{\alpha} \nabla \tilde{\mathbf{v}}_{\alpha}\right) \mathrm{d} A \quad \alpha=\beta, \gamma
\end{aligned}
$$

Bearing in mind Eq. 39, it must be noticed that the closure equation for each phase remains valid under the associated time-scale constraint that can be recalled in the form

$$
t^{*} \gg \max \left(\max _{\alpha=\beta, \gamma}\left(\frac{\rho_{\alpha} l_{\alpha}^{2}}{\mu_{\alpha}}\right), \max _{\alpha=\beta, \gamma}\left(\frac{l_{\alpha}}{\left\|\left\langle\mathbf{v}_{\alpha}\right\rangle^{\alpha}\right\|}\right)\right)
$$

Since

$$
0 \leq l_{\alpha} \leq l_{p}
$$

\footnotetext{
1 The consequence of this time-scale constraint is that (i) the acceleration term could have been neglected in comparison to the viscous term in the point momentum balance equations, and (ii) the non-stationary term in the macroscopic momentum balance equations will be negligible as will be seen later.
} 
where $l_{p}$ designates the mean pore size of the medium, a safe but more useful version of the constraint given by Eq. 55 can be expressed as ${ }^{2}$

$$
t^{*} \gg l_{p} \max \left(l_{p} \max _{\alpha=\beta, \gamma}\left(\frac{\rho_{\alpha}}{\mu_{\alpha}}\right), \max _{\alpha=\beta, \gamma}\left(\frac{\varepsilon_{\alpha}}{\left\|\left\langle\mathbf{v}_{\alpha}\right\rangle\right\|}\right)\right)
$$

To complete the closure problem, we now need to construct boundary conditions in order to state the boundary value problem for the pressure and velocity spatial deviations and this is the object of the next paragraph.

\subsection{Boundary Conditions}

By making use of the velocity and pressure decompositions given by Eqs. 20 and 27, we can express Eqs. 4-6 as

$$
\begin{gathered}
\text { BC1 and BC2 } \quad \tilde{\mathbf{v}}_{\alpha}=-\left\langle\mathbf{v}_{\alpha}\right\rangle^{\alpha} \quad \text { at } A_{\alpha \sigma}, \quad \alpha=\beta \text { and } \gamma \\
\text { BC3 } \quad \tilde{\mathbf{v}}_{\beta}=\tilde{\mathbf{v}}_{\gamma}-\left(\left\langle\mathbf{v}_{\beta}\right\rangle^{\beta}-\left\langle\mathbf{v}_{\gamma}\right\rangle^{\gamma}\right) \quad \text { at } A_{\beta \gamma} \\
\mathrm{BC} 4 \quad-\mathbf{n}_{\beta \gamma} \tilde{p}_{\beta}=-\mathbf{n}_{\beta \gamma} \tilde{p}_{\gamma}+\mathbf{n}_{\beta \gamma}\left(\left\langle p_{\beta}\right\rangle^{\beta}-\left\langle p_{\gamma}\right\rangle^{\gamma}\right) \\
-\mathbf{n}_{\beta \gamma} \cdot\left[\mu_{\beta}\left(\nabla \tilde{\mathbf{v}}_{\beta}+\nabla \tilde{\mathbf{v}}_{\beta}^{T}\right)-\mu_{\gamma}\left(\nabla \tilde{\mathbf{v}}_{\gamma}+\nabla \tilde{\mathbf{v}}_{\gamma}^{T}\right)\right]+2 \sigma H \mathbf{n}_{\beta \gamma} \quad \text { at } A_{\beta \gamma}
\end{gathered}
$$

In deriving Eq. 58c we have used the representation of the viscous stress tensors, $\mathbf{T}_{v \alpha}$ ( $\alpha=\beta, \gamma$ ), according to Eq. 9. Moreover, we have discarded the macroscopic viscous part of these two tensors. This is a straightforward consequence of the scale hierarchy described by Eq. 16 considering the fact that the characteristic length-scale of the gradient is $l_{\beta}$ or $l_{\gamma}$ when operating on the velocity deviation and $L$ when operating on the average part of the velocity.

Under this form, many source terms appear in the set of boundary conditions, namely $\left\langle\mathbf{v}_{\beta}\right\rangle^{\beta},\left\langle\mathbf{v}_{\gamma}\right\rangle^{\gamma},\left\langle p_{\beta}\right\rangle^{\beta}-\left\langle p_{\gamma}\right\rangle^{\gamma}$ and $2 \sigma H$ leading to an excessively complex closure. A similar issue was discussed thoroughly in the derivation of macroscopic models for two-phase creeping flow in porous media (Whitaker 1986b; Torres 1987; Whitaker 1994) involving the same boundary conditions. To achieve necessary simplifications, it is convenient to begin with the surface average over $A_{\beta \gamma}$ of the projection of Eq. $58 \mathrm{c}$ onto the normal $\mathbf{n}_{\beta \gamma}$. The result can be listed as (Whitaker 1994)

$$
\begin{aligned}
-\left(\left\langle p_{\beta}\right\rangle^{\beta}-\left\langle p_{\gamma}\right\rangle^{\gamma}\right)= & 2 \sigma\langle H\rangle_{\beta \gamma}+\left\langle\tilde{p}_{\beta}-\tilde{p}_{\gamma}\right\rangle_{\beta \gamma} \\
& -\left\langle\mathbf{n}_{\beta \gamma} \cdot\left[\mu_{\beta}\left(\nabla \tilde{\mathbf{v}}_{\beta}+\nabla \tilde{\mathbf{v}}_{\beta}^{T}\right)-\mu_{\gamma}\left(\nabla \tilde{\mathbf{v}}_{\gamma}+\nabla \tilde{\mathbf{v}}_{\gamma}^{T}\right)\right] \cdot \mathbf{n}_{\beta \gamma}\right\rangle_{\beta \gamma}
\end{aligned}
$$

where the area average is denoted by \langle\rangle$_{\beta \gamma}$ and where we have considered $\left\langle p_{\beta}\right\rangle^{\beta}-\left\langle p_{\gamma}\right\rangle^{\gamma}$ as a constant with respect to that averaging process following arguments similar to those leading to Eq. 21. If the pressure, viscous and inertial forces are of comparable magnitude it can be deduced from Eq. 54 that

$$
\begin{aligned}
\tilde{p}_{\alpha} & =O\left(\mu_{\alpha} l_{\alpha}\left\|\nabla^{2} \tilde{\mathbf{v}}_{\alpha}\right\|\right)+O\left(\rho_{\alpha} l_{\alpha}\left\|\mathbf{v}_{\alpha} \cdot \nabla \tilde{\mathbf{v}}_{\alpha}\right\|\right) \\
& =O\left(\mu_{\alpha}\left\|\nabla \tilde{\mathbf{v}}_{\alpha}\right\|\right)+O\left(\rho_{\alpha} l_{\alpha}\left\|\mathbf{v}_{\alpha} \cdot \nabla \tilde{\mathbf{v}}_{\alpha}\right\|\right), \quad \alpha=\beta, \gamma
\end{aligned}
$$

\footnotetext{
2 A particular situation where this constraint is satisfied is that of an entirely time independent two-phase flow process. It can be envisaged for many problems of practical importance such as the co-injection (co-current or counter-current flow) of two-phases over sufficiently large periods of time, as encountered for instance in packed bed columns of reactors. Note also that, in this particular case, the average mass balance equations simplify to stationary versions $\nabla \cdot\left\langle\mathbf{v}_{\beta}\right\rangle=0$ and $\nabla \cdot\left\langle\mathbf{v}_{\gamma}\right\rangle=0$. Again, this situation is only a limit case of processes envisaged here.
} 
Using this allows us to write

$$
-\left(\left\langle p_{\beta}\right\rangle^{\beta}-\left\langle p_{\gamma}\right\rangle^{\gamma}\right)=2 \sigma\langle H\rangle_{\beta \gamma}+\max _{\alpha=\beta, \gamma}\left(O\left\langle\mu_{\alpha} \nabla \tilde{\mathbf{v}}_{\alpha}\right\rangle_{\beta \gamma}+O\left\langle\rho_{\alpha} l_{\alpha} \mathbf{v}_{\alpha} \cdot \nabla \tilde{\mathbf{v}}_{\alpha}\right\rangle_{\beta \gamma}\right)
$$

or

$$
\begin{aligned}
- & \left(\left\langle p_{\beta}\right\rangle^{\beta}-\left\langle p_{\gamma}\right\rangle^{\gamma}\right)=2 \sigma\langle H\rangle_{\beta \gamma} \\
& \times\left(1+\max _{\alpha=\beta, \gamma}\left(O\left(\frac{\left\langle\left\|\nabla \tilde{\mathbf{v}}_{\alpha}\right\|\right\rangle_{\beta \gamma}}{\langle H\rangle_{\beta \gamma}\left\|\left\langle\mathbf{v}_{\alpha}\right\rangle^{\alpha}\right\|}\right) C a_{\alpha}+O\left(\frac{\left\langle\left\|\mathbf{v}_{\alpha} \cdot \nabla \tilde{\mathbf{v}}_{\alpha}\right\|\right\rangle_{\beta \gamma}}{\langle H\rangle_{\beta \gamma}\left\|\left\langle\mathbf{v}_{\alpha}\right\rangle^{\alpha}\right\|^{2}}\right) W e_{\alpha}\right)\right)
\end{aligned}
$$

In this last equation, $C a_{\alpha}$ and $W e_{\alpha}$ are the capillary number and Weber number in the $\alpha$-phase, respectively, defined as

$$
C a_{\alpha}=\frac{\mu_{\alpha}\left\|\left\langle\mathbf{v}_{\alpha}\right\rangle^{\alpha}\right\|}{\sigma}
$$

and

$$
W e_{\alpha}=\frac{\rho_{\alpha}\left\|\left\langle\mathbf{v}_{\alpha}\right\rangle^{\alpha}\right\|^{2} l_{p}}{\sigma}
$$

where we have used $l_{p}$ to represent the mean pore size. We now impose the restrictions

$$
\left(\frac{\left\langle\left\|\nabla \tilde{\mathbf{v}}_{\alpha}\right\|\right\rangle_{\beta \gamma}}{\langle H\rangle_{\beta \gamma}\left\|\left\langle\mathbf{v}_{\alpha}\right\rangle^{\alpha}\right\|}\right) C a_{\alpha} \ll 1, \quad \alpha=\beta, \gamma
$$

and

$$
\left(\frac{\left\langle\left\|\mathbf{v}_{\alpha} \cdot \nabla \tilde{\mathbf{v}}_{\alpha}\right\|\right\rangle_{\beta \gamma}}{\langle H\rangle_{\beta \gamma}\left\|\left\langle\mathbf{v}_{\alpha}\right\rangle^{\alpha}\right\|^{2}}\right) W e_{\alpha} \ll 1, \quad \alpha=\beta, \gamma
$$

so that we can write Eq. 62 as

$$
-\left(\left\langle p_{\beta}\right\rangle^{\beta}-\left\langle p_{\gamma}\right\rangle^{\gamma}\right)=2 \sigma\langle H\rangle_{\beta \gamma}
$$

which is nothing else than the usual capillary pressure. If we impose that $\langle H\rangle_{\beta \gamma}$ is at most $O\left(l_{p}^{-1}\right)$ which is a reasonable approximation if the solid phase is wetted by one of the two fluid phases, and neither the $\beta$ - nor the the $\gamma$-phase is under the form of ganglia of radius significantly smaller than the mean pore size, then one can reasonably expect

$$
\frac{\left\langle\left\|\nabla \tilde{\mathbf{v}}_{\alpha}\right\|\right\rangle_{\beta \gamma}}{\langle H\rangle_{\beta \gamma}\left\|\left\langle\mathbf{v}_{\alpha}\right\rangle^{\alpha}\right\|} \ll 1, \quad \alpha=\beta, \gamma
$$

and

$$
\frac{\left\langle\left\|\mathbf{v}_{\alpha} \cdot \nabla \tilde{\mathbf{v}}_{\alpha}\right\|\right\rangle_{\beta \gamma}}{\langle H\rangle_{\beta \gamma}\left\|\left\langle\mathbf{v}_{\alpha}\right\rangle^{\alpha}\right\|^{2}} \ll 1, \quad \alpha=\beta, \gamma
$$

This can be readily inferred from the fact that $\left\langle\left\|\nabla \tilde{\mathbf{v}}_{\alpha}\right\|\right\rangle_{\beta \gamma}$ and $\left\langle\left\|\mathbf{v}_{\alpha} \cdot \nabla \tilde{\mathbf{v}}_{\alpha}\right\|\right\rangle_{\beta \gamma}$ are expected to be much smaller in magnitude than $\frac{\left\|\left\langle\mathbf{v}_{\alpha}\right\rangle^{\alpha}\right\|}{l_{p}}$ and $\frac{\left\|\left\langle\mathbf{v}_{\alpha}\right\rangle^{\alpha}\right\|^{2}}{l_{p}}$, respectively. Under these circumstances, one would only require $C a_{\alpha}$ and $W e_{\alpha}$ to be at most of order 1 for the above development to hold. Nevertheless, one must keep in mind that the requirements are those 
given by Eqs. 65 and 66 and when these constraints are satisfied, the stress jump at $A_{\beta \gamma}$ can be written as (Torres 1987)

$$
\begin{aligned}
\mathrm{BC} 3-\mathbf{n}_{\beta \gamma} \tilde{p}_{\beta}= & -\mathbf{n}_{\beta \gamma} \tilde{p}_{\gamma}-\mathbf{n}_{\beta \gamma} \cdot\left[\mu_{\beta}\left(\nabla \tilde{\mathbf{v}}_{\beta}+\nabla \tilde{\mathbf{v}}_{\beta}^{T}\right)-\mu_{\gamma}\left(\nabla \tilde{\mathbf{v}}_{\gamma}+\nabla \tilde{\mathbf{v}}_{\gamma}^{T}\right)\right] \\
& +2 \sigma\left(H-\langle H\rangle_{\beta \gamma}\right) \mathbf{n}_{\beta \gamma} \quad \text { at } A_{\beta \gamma}
\end{aligned}
$$

At this point, the closure still involves the term of curvature deviation $H-\langle H\rangle_{\beta \gamma}$ in the above boundary condition. Conditions under which this term can exhibit significant variations over the averaging volume are not easy to identify due the diversity of geometrical, wetting and hydrodynamic situations that can be encountered. Clearly, comprehensive numerical experiments of two-phase flow in complex configurations would greatly highlight this issue and provide some explicit criteria on the importance of this term. In the absence of more detailed information on that matter, we shall follow an assumption suggested in previous studies on two-phase creeping flow (Auriault 1987; Whitaker 1994; Lasseux et al. 1996) which consists of neglecting the term involving the curvature deviation in comparison to the pressure and viscous deviation terms in Eq. 70. Physically, this means that, at the closure level only, capillary effects are negligible. One of the necessary conditions to put forth such an hypothesis certainly lies in negligible gravity effects over $V$ in comparison to capillary effects, leading to a small pore-scale Bond number compared to unity. It is however highly probable that this is not a sufficient requirement. In the rest of this work, we keep the hypothesis of negligible capillary effects in the closure.

Since the objective is not to solve the boundary value problem for the closure over the entire macroscopic structure, we assume that a representative elementary volume can be exhibited on that structure. This allows one to replace the deviation form of the boundary condition expressed in Eq. 7 by a periodic one leading to a local form of the closure problem that is written as

$$
\begin{aligned}
\rho_{\alpha} \mathbf{v}_{\alpha} \cdot \nabla \tilde{\mathbf{v}}_{\alpha}= & -\nabla \tilde{p}_{\alpha}+\mu_{\alpha} \nabla^{2} \tilde{\mathbf{v}}_{\alpha} \\
& -\frac{1}{V_{\alpha}} \int_{A_{\alpha \sigma} \cup A_{\beta \gamma}} \mathbf{n}_{\alpha} \cdot\left(-\tilde{p}_{\alpha} \mathbf{I}+\mu_{\alpha} \nabla \tilde{\mathbf{v}}_{\alpha}\right) \mathrm{d} A \quad \text { in } V_{\alpha}, \quad \alpha=\beta \text { and } \gamma \\
& \nabla \cdot \tilde{\mathbf{v}}_{\alpha}=0 \quad \text { in } V_{\alpha}, \quad \alpha=\beta \text { and } \gamma \\
& \text { BC1 and BC2 } \quad \tilde{\mathbf{v}}_{\alpha}=-\left\langle\mathbf{v}_{\alpha}\right\rangle^{\alpha} \text { at } A_{\alpha \sigma}, \quad \alpha=\beta \text { and } \gamma \\
& \text { BC3 } \tilde{\mathbf{v}}_{\beta}=\tilde{\mathbf{v}}_{\gamma}-\left(\left\langle\mathbf{v}_{\beta}\right\rangle^{\beta}-\left\langle\mathbf{v}_{\gamma}\right\rangle^{\gamma}\right) \text { at } A_{\beta \gamma} \\
& \text { BC4 }-\mathbf{n}_{\beta \gamma} \tilde{p}_{\beta}=-\mathbf{n}_{\beta \gamma} \tilde{p}_{\gamma} \\
& -\mathbf{n}_{\beta \gamma} \cdot\left[\mu_{\beta}\left(\nabla \tilde{\mathbf{v}}_{\beta}+\nabla \tilde{\mathbf{v}}_{\beta}^{T}\right)-\mu_{\gamma}\left(\nabla \tilde{\mathbf{v}}_{\gamma}+\nabla \tilde{\mathbf{v}}_{\gamma}^{T}\right)\right] \quad \text { at } A_{\beta \gamma}
\end{aligned}
$$

Periodicity

$$
\begin{aligned}
& \tilde{\mathbf{v}}_{\alpha}\left(\mathbf{r}+\mathbf{l}_{i}\right)=\tilde{\mathbf{v}}_{\alpha}(\mathbf{r}) \quad \alpha=\beta \text { and } \gamma \quad i=1,2,3 \\
& \tilde{p}_{\alpha}\left(\mathbf{r}+\mathbf{l}_{i}\right)=\tilde{p}_{\alpha}(\mathbf{r}) \quad \alpha=\beta \text { and } \gamma \quad i=1,2,3
\end{aligned}
$$

Average

$$
\left\langle\tilde{\mathbf{v}}_{\alpha}\right\rangle^{\alpha}=0 \quad \alpha=\beta \text { and } \gamma
$$

This last condition, as expressed in Eq. 22, is part of the development in addition to the time-scale constraint of Eq. 55 and the hypothesis of negligible capillary effects at the closure level. 
An equivalent condition to that given in Eq. 10 would be required to avoid the solution on $\tilde{p}_{\beta}$ and $\tilde{p}_{\gamma}$ to within an arbitrary additive constant. This could be obtained by a condition on one of the pressure deviations. However, this condition is of no practical importance since any additive constant in the pressure deviation fields does not affect the macroscopic closed form of the governing equations. For this reason, no such condition will be considered in our development.

Even if no explicit dependence of the curvature appears in the closure problem, one must keep in mind that the interface $A_{\beta \gamma}$ must be located before the solution of the set of equations 71.

\subsection{Closure Variables}

The local closure problem given by Eq. 71 is non-homogeneous because of the presence of the two source terms $\left\langle\mathbf{v}_{\beta}\right\rangle^{\beta}$ and $\left\langle\mathbf{v}_{\gamma}\right\rangle^{\gamma}$. For this reason, we want to find a solution of the closure in terms of these sources leading to a representation for the spatial deviation velocities and pressures given by ${ }^{3}$

$$
\begin{aligned}
& \tilde{\mathbf{v}}_{\alpha}=\mathbf{A}_{\alpha \beta} \cdot\left\langle\mathbf{v}_{\beta}\right\rangle^{\beta}+\mathbf{A}_{\alpha \gamma} \cdot\left\langle\mathbf{v}_{\gamma}\right\rangle^{\gamma} \quad \alpha=\beta, \gamma \\
& \tilde{p}_{\alpha}=\mu_{\alpha}\left[\mathbf{a}_{\alpha \beta} \cdot\left\langle\mathbf{v}_{\beta}\right\rangle^{\beta}+\mathbf{a}_{\alpha \gamma} \cdot\left\langle\mathbf{v}_{\gamma}\right\rangle^{\gamma}\right] \quad \alpha=\beta, \gamma
\end{aligned}
$$

In this representation, $\mathbf{A}_{\alpha \kappa}$ and $\mathbf{a}_{\alpha \kappa}$ are tensors and vectors referred to as closure variables with the nomenclature arranged so that $\alpha$ represents the phase in which the closure variable is defined and $\kappa$ indicates which velocity is mapped onto the spatial deviation. This representation can now be inserted in the closure problem provided above and we choose to specify the closure variables according to two boundary value problems each one being associated to one of the source terms. For the coefficients associated to $\left\langle\mathbf{v}_{\beta}\right\rangle^{\beta}$, we obtain

Problem I:

$$
\begin{aligned}
& \left(\rho_{\alpha} \mathbf{v}_{\alpha} / \mu_{\alpha}\right) \cdot \nabla \mathbf{A}_{\alpha \beta}=-\nabla \mathbf{a}_{\alpha \beta}+\nabla^{2} \mathbf{A}_{\alpha \beta} \\
& -\frac{1}{V_{\alpha}} \int_{A_{\alpha \sigma} \cup A_{\beta \gamma}} \mathbf{n}_{\alpha} \cdot\left(-\mathbf{I} \mathbf{a}_{\alpha \beta}+\nabla \mathbf{A}_{\alpha \beta}\right) \mathrm{d} A \quad \text { in } V_{\alpha}, \quad \alpha=\beta \text { and } \gamma \\
& \nabla \cdot \mathbf{A}_{\alpha \beta}=0 \quad \text { in } V_{\alpha}, \quad \alpha=\beta \text { and } \gamma \\
& \mathrm{BC} 1 \text { and } \mathrm{BC} 2 \quad \mathbf{A}_{\alpha \beta}=-\delta_{\alpha \beta} \mathbf{I} \quad \text { at } A_{\alpha \sigma}, \quad \alpha=\beta \text { and } \gamma \\
& \text { BC3 } \quad \mathbf{A}_{\beta \beta}=\mathbf{A}_{\gamma \beta}-\mathbf{I} \quad \text { at } A_{\beta \gamma} \\
& \text { BC4 } \mu_{\beta} \mathbf{n}_{\beta \gamma} \cdot\left[-\mathbf{I} \mathbf{a}_{\beta \beta}+\left(\nabla \mathbf{A}_{\beta \beta}+\nabla \mathbf{A}_{\beta \beta}^{T}\right)\right] \\
& =\mu_{\gamma} \mathbf{n}_{\beta \gamma} \cdot\left[-\mathbf{I a}_{\gamma \beta}+\left(\nabla \mathbf{A}_{\gamma \beta}+\nabla \mathbf{A}_{\gamma \beta}^{T}\right)\right] \quad \text { at } A_{\beta \gamma}
\end{aligned}
$$

Periodicity

$$
\begin{array}{rll}
\mathbf{a}_{\alpha \beta}\left(\mathbf{r}+\mathbf{l}_{i}\right)=\mathbf{a}_{\alpha \beta}(\mathbf{r}) & \alpha=\beta \text { and } \gamma & i=1,2,3 \\
\mathbf{A}_{\alpha \beta}\left(\mathbf{r}+\mathbf{l}_{i}\right)=\mathbf{A}_{\alpha \beta}(\mathbf{r}) & \alpha=\beta \text { and } \gamma & i=1,2,3
\end{array}
$$

Average

$$
\left\langle\mathbf{A}_{\alpha \beta}\right\rangle^{\alpha}=0 \quad \alpha=\beta \text { and } \gamma
$$

\footnotetext{
3 In this mapping, we have omitted additive scalar and vector fields since it can be proven, following the same development as the one provided for creeping two-phase flow (Whitaker 1986b), that these fields are either zero or constants of no importance in the macroscopic governing equations.
} 
Here, we have used the conventional notation $\delta_{\alpha \beta}$ for the Kronecker delta function.

The second boundary value problem associated with the coefficients of $\left\langle\mathbf{v}_{\gamma}\right\rangle^{\gamma}$ takes the analogous form given by

Problem II:

$$
\begin{aligned}
& \left(\rho_{\alpha} \mathbf{v}_{\alpha} / \mu_{\alpha}\right) \cdot \nabla \mathbf{A}_{\alpha \gamma}=-\nabla \mathbf{a}_{\alpha \gamma}+\nabla^{2} \mathbf{A}_{\alpha \gamma} \\
& -\frac{1}{V_{\alpha}} \int_{A_{\alpha \sigma} \cup A_{\beta \gamma}} \mathbf{n}_{\alpha} \cdot\left(-\mathbf{I} \mathbf{a}_{\alpha \gamma}+\nabla \mathbf{A}_{\alpha \gamma}\right) \mathrm{d} A \quad \text { in } V_{\alpha}, \quad \alpha=\beta \text { and } \gamma \\
& \nabla \cdot \mathbf{A}_{\alpha \gamma}=0 \quad \text { in } V_{\alpha}, \quad \alpha=\beta \text { and } \gamma \\
& \mathrm{BC} 1 \text { and BC2 } \quad \mathbf{A}_{\alpha \gamma}=-\delta_{\alpha \gamma} \mathbf{I} \text { at } A_{\alpha \sigma}, \quad \alpha=\beta \text { and } \gamma \\
& \mathrm{BC} 3 \quad \mathbf{A}_{\beta \gamma}=\mathbf{A}_{\gamma \gamma}+\mathbf{I} \quad \text { at } A_{\beta \gamma} \\
& \mathrm{BC} 4 \quad \mu_{\beta} \mathbf{n}_{\beta \gamma} \cdot\left[-\mathbf{I} \mathbf{a}_{\beta \gamma}+\left(\nabla \mathbf{A}_{\beta \gamma}+\nabla \mathbf{A}_{\beta \gamma}^{T}\right)\right] \\
& =\mu_{\gamma} \mathbf{n}_{\beta \gamma} \cdot\left[-\mathbf{I} \mathbf{a}_{\gamma \gamma}+\left(\nabla \mathbf{A}_{\gamma \gamma}+\nabla \mathbf{A}_{\gamma \gamma}^{T}\right)\right] \text { at } A_{\beta \gamma}
\end{aligned}
$$

Periodicity

$$
\begin{aligned}
& \mathbf{a}_{\alpha \gamma}\left(\mathbf{r}+\mathbf{l}_{i}\right)=\mathbf{a}_{\alpha \gamma}(\mathbf{r}) \quad \alpha=\beta \text { and } \gamma \quad i=1,2,3 \\
& \mathbf{A}_{\alpha \gamma}\left(\mathbf{r}+\mathbf{l}_{i}\right)=\mathbf{A}_{\alpha \gamma}(\mathbf{r}) \quad \alpha=\beta \text { and } \gamma \quad i=1,2,3
\end{aligned}
$$

Average

$$
\left\langle\mathbf{A}_{\alpha \gamma}\right\rangle^{\alpha}=0 \quad \alpha=\beta \text { and } \gamma
$$

\subsection{Decomposition of the Closure Problems}

We shall progress towards some more tractable versions of the above two boundary value problems by using a convenient decomposition with the idea that this decomposition will lead to a macroscopic model containing the classical Darcy part and a remaining part containing the inertial effects. To accomplish this, we decompose the four tensors and four vectors defined in Eqs. 72 and 73 according to

$$
\begin{aligned}
& \mathbf{A}_{\alpha \kappa}=\mathbf{A}_{\alpha \kappa 1}+\mathbf{A}_{\alpha \kappa 2} \quad \alpha, \kappa=\beta, \gamma \\
& \mathbf{a}_{\alpha \kappa}=\mathbf{a}_{\alpha \kappa 1}+\mathbf{a}_{\alpha \kappa 2} \quad \alpha, \kappa=\beta, \gamma
\end{aligned}
$$

and we choose to specify the tensors and vectors $\mathbf{A}_{\beta \beta 1}, \mathbf{a}_{\beta \beta 1}, \mathbf{A}_{\gamma \beta 1}$ and $\mathbf{a}_{\gamma \beta 1}$ by the following problem

Problem I1

$$
\begin{aligned}
& -\nabla \mathbf{a}_{\alpha \beta 1}+\nabla^{2} \mathbf{A}_{\alpha \beta 1} \\
& =-\varepsilon_{\beta} \mathbf{K}_{\alpha}^{-1} \cdot\left[\delta_{\alpha \beta} \mathbf{I}+\left(\delta_{\alpha \beta}-1\right) \mathbf{K}_{\gamma \beta}\right] \quad \text { in } V_{\alpha}, \quad \alpha=\beta \text { and } \gamma \\
& \nabla \cdot \mathbf{A}_{\alpha \beta 1}=0 \quad \text { in } V_{\alpha}, \quad \alpha=\beta \text { and } \gamma \\
& \text { BC1 and BC2 } \quad \mathbf{A}_{\alpha \beta 1}=-\delta_{\alpha \beta} \mathbf{I} \quad \text { at } A_{\alpha \sigma}, \quad \alpha=\beta \text { and } \gamma \\
& \text { BC3 } \quad \mathbf{A}_{\beta \beta 1}=\mathbf{A}_{\gamma \beta 1}-\mathbf{I} \quad \text { at } A_{\beta \gamma} \\
& \text { BC4 } \quad \mu_{\beta} \mathbf{n}_{\beta \gamma} \cdot\left[-\mathbf{I} \mathbf{a}_{\beta \beta 1}+\left(\nabla \mathbf{A}_{\beta \beta 1}+\nabla \mathbf{A}_{\beta \beta 1}^{T}\right)\right] \\
& =\mu_{\gamma} \mathbf{n}_{\beta \gamma} \cdot\left[-\mathbf{I} \mathbf{a}_{\gamma \beta 1}+\left(\nabla \mathbf{A}_{\gamma \beta 1}+\nabla \mathbf{A}_{\gamma \beta 1}^{T}\right)\right] \text { at } A_{\beta \gamma}
\end{aligned}
$$


Periodicity

$$
\begin{aligned}
& \mathbf{a}_{\alpha \beta 1}\left(\mathbf{r}+\mathbf{l}_{i}\right)=\mathbf{a}_{\alpha \beta 1}(\mathbf{r}) \quad \alpha=\beta \text { and } \gamma \quad i=1,2,3 \\
& \mathbf{A}_{\alpha \beta 1}\left(\mathbf{r}+\mathbf{l}_{i}\right)=\mathbf{A}_{\alpha \beta 1}(\mathbf{r}) \quad \alpha=\beta \text { and } \gamma \quad i=1,2,3
\end{aligned}
$$

Average

$$
\left\langle\mathbf{A}_{\alpha \beta 1}\right\rangle^{\alpha}=0 \quad \alpha=\beta \text { and } \gamma
$$

Similarly, we specify the tensors and vectors $\mathbf{A}_{\beta \gamma 1}, \mathbf{a}_{\beta \gamma 1}, \mathbf{A}_{\gamma \gamma 1}$ and $\mathbf{a}_{\gamma \gamma 1}$ by the following problem

Problem II1

$$
\begin{aligned}
& -\nabla \mathbf{a}_{\alpha \gamma 1}+\nabla^{2} \mathbf{A}_{\alpha \gamma 1} \\
& =-\varepsilon_{\gamma} \mathbf{K}_{\alpha}^{-1} \cdot\left[\delta_{\alpha \gamma} \mathbf{I}+\left(\delta_{\alpha \gamma}-1\right) \mathbf{K}_{\beta \gamma}\right] \quad \text { in } V_{\alpha}, \quad \alpha=\beta \text { and } \gamma \\
& \nabla \cdot \mathbf{A}_{\alpha \gamma 1}=0 \text { in } V_{\alpha}, \quad \alpha=\beta \text { and } \gamma \\
& \text { BC1 and BC2 } \quad \mathbf{A}_{\alpha \gamma 1}=-\delta_{\alpha \gamma} \mathbf{I} \quad \text { at } A_{\alpha \sigma}, \quad \alpha=\beta \text { and } \gamma \\
& \text { BC3 } \quad \mathbf{A}_{\beta \gamma 1}=\mathbf{A}_{\gamma \gamma 1}+\mathbf{I} \quad \text { at } A_{\beta \gamma} \\
& \text { BC4 } \quad \mu_{\beta} \mathbf{n}_{\beta \gamma} \cdot\left[-\mathbf{I} \mathbf{a}_{\beta \gamma_{1}}+\left(\nabla \mathbf{A}_{\beta \gamma 1}+\nabla \mathbf{A}_{\beta \gamma 1}^{T}\right)\right] \\
& =\mu_{\gamma} \mathbf{n}_{\beta \gamma} \cdot\left[-\mathbf{I} \mathbf{a}_{\gamma \gamma_{1}}+\left(\nabla \mathbf{A}_{\gamma \gamma 1}+\nabla \mathbf{A}_{\gamma \gamma 1}^{T}\right)\right] \text { at } A_{\beta \gamma}
\end{aligned}
$$

Periodicity

$$
\begin{aligned}
& \mathbf{a}_{\alpha \gamma 1}\left(\mathbf{r}+\mathbf{l}_{i}\right)=\mathbf{a}_{\alpha \gamma 1}(\mathbf{r}) \quad \alpha=\beta \text { and } \gamma \quad i=1,2,3 \\
& \mathbf{A}_{\alpha \gamma 1}\left(\mathbf{r}+\mathbf{l}_{i}\right)=\mathbf{A}_{\alpha \gamma 1}(\mathbf{r}) \quad \alpha=\beta \text { and } \gamma \quad i=1,2,3
\end{aligned}
$$

Average

$$
\left\langle\mathbf{A}_{\alpha \gamma 1}\right\rangle^{\alpha}=0 \quad \alpha=\beta \text { and } \gamma
$$

While writing Eqs. 78a and 79a we have used the following definitions

$$
\begin{gathered}
\frac{1}{V_{\alpha}} \int_{A_{\alpha \sigma} \cup A_{\beta \gamma}} \mathbf{n}_{\alpha} \cdot\left(-\mathbf{I} \mathbf{a}_{\alpha \alpha 1}+\nabla \mathbf{A}_{\alpha \alpha 1}\right) \mathrm{d} A=-\varepsilon_{\alpha} \mathbf{K}_{\alpha}^{-1} \quad \alpha=\beta, \gamma \\
\frac{1}{V_{\alpha}} \int_{A_{\alpha \sigma} \cup A_{\beta \gamma}} \mathbf{n}_{\alpha} \cdot\left(-\mathbf{I a}_{\alpha \kappa 1}+\nabla \mathbf{A}_{\alpha \kappa 1}\right) \mathrm{d} A=\varepsilon_{\kappa} \mathbf{K}_{\alpha}^{-1} \cdot \mathbf{K}_{\alpha \kappa} \quad \alpha, \kappa=\beta, \gamma, \quad \alpha \neq \kappa
\end{gathered}
$$

where $\mathbf{K}_{\alpha}$ and $\mathbf{K}_{\alpha \kappa}$ are the permeability and viscous drag tensors as used in the derivation of the macroscopic two-phase flow model in the creeping regime (Whitaker 1986b, 1994; Lasseux et al. 1996). The closure problem must now be completed with the two following boundary value problems defining the four tensors $\mathbf{A}_{\beta \beta 2}, \mathbf{A}_{\gamma \beta 2}, \mathbf{A}_{\beta \gamma 2}$ and $\mathbf{A}_{\gamma \gamma 2}$ and the four vectors $\mathbf{a}_{\beta \beta 2}, \mathbf{a}_{\gamma \beta 2}, \mathbf{a}_{\beta \gamma 2}$ and $\mathbf{a}_{\gamma \gamma 2}$ 
Problem I2

$$
\begin{aligned}
& \left(\rho_{\alpha} \mathbf{v}_{\alpha} / \mu_{\alpha}\right) \cdot \nabla \mathbf{A}_{\alpha \beta 1}+\left(\rho_{\alpha} \mathbf{v}_{\alpha} / \mu_{\alpha}\right) \cdot \nabla \mathbf{A}_{\alpha \beta 2} \\
& =-\nabla \mathbf{a}_{\alpha \beta 2}+\nabla^{2} \mathbf{A}_{\alpha \beta 2}+\varepsilon_{\beta} \mathbf{K}_{\alpha}^{-1} \cdot \mathbf{F}_{\alpha \beta} \quad \text { in } V_{\alpha}, \quad \alpha=\beta \text { and } \gamma \\
& \nabla \cdot \mathbf{A}_{\alpha \beta 2}=0 \quad \text { in } V_{\alpha}, \quad \alpha=\beta \text { and } \gamma \\
& \text { BC1 and BC2 } \quad \mathbf{A}_{\alpha \beta 2}=0 \quad \text { at } A_{\alpha \sigma}, \quad \alpha=\beta \text { and } \gamma \\
& \text { BC3 } \quad \mathbf{A}_{\beta \beta 2}=\mathbf{A}_{\gamma \beta 2} \text { at } A_{\beta \gamma} \\
& \text { BC4 } \quad \mu_{\beta} \mathbf{n}_{\beta \gamma} \cdot\left[-\mathbf{I} \mathbf{a}_{\beta \beta 2}+\left(\nabla \mathbf{A}_{\beta \beta 2}+\nabla \mathbf{A}_{\beta \beta 2}^{T}\right)\right] \\
& =\mu_{\gamma} \mathbf{n}_{\beta \gamma} \cdot\left[-\mathbf{I} \mathbf{a}_{\gamma \beta 2}+\left(\nabla \mathbf{A}_{\gamma \beta 2}+\nabla \mathbf{A}_{\gamma \beta 2}^{T}\right)\right] \text { at } A_{\beta \gamma}
\end{aligned}
$$

Periodicity

$$
\begin{aligned}
\mathbf{A}_{\alpha \beta 2}\left(\mathbf{r}+\mathbf{l}_{i}\right) & =\mathbf{A}_{\alpha \beta 2}(\mathbf{r}) \quad \alpha=\beta \text { and } \gamma \quad i=1,2,3 \\
\mathbf{a}_{\alpha \beta 2}\left(\mathbf{r}+\mathbf{l}_{i}\right) & =\mathbf{a}_{\alpha \beta 2}(\mathbf{r}) \quad \alpha=\beta \text { and } \gamma \quad i=1,2,3
\end{aligned}
$$

Average

$$
\left\langle\mathbf{A}_{\alpha \beta 2}\right\rangle^{\alpha}=0 \quad \alpha=\beta \text { and } \gamma
$$

Problem II2

$$
\begin{aligned}
& \left(\rho_{\alpha} \mathbf{v}_{\alpha} / \mu_{\alpha}\right) \cdot \nabla \mathbf{A}_{\alpha \gamma 1}+\left(\rho_{\alpha} \mathbf{v}_{\alpha} / \mu_{\alpha}\right) \cdot \nabla \mathbf{A}_{\alpha \gamma 2} \\
& =-\nabla \mathbf{a}_{\alpha \gamma 2}+\nabla^{2} \mathbf{A}_{\alpha \gamma 2}+\varepsilon_{\gamma} \mathbf{K}_{\alpha}^{-1} \cdot \mathbf{F}_{\alpha \gamma} \quad \text { in } V_{\alpha}, \quad \alpha=\beta \text { and } \gamma \\
& \nabla \cdot \mathbf{A}_{\alpha \gamma 2}=0 \quad \text { in } V_{\alpha}, \quad \alpha=\beta \text { and } \gamma \\
& \text { BC1 and BC2 } \quad \mathbf{A}_{\alpha \gamma 2}=0 \quad \text { at } A_{\alpha \sigma}, \quad \alpha=\beta \text { and } \gamma \\
& \text { BC3 } \quad \mathbf{A}_{\beta \gamma 2}=\mathbf{A}_{\gamma \gamma 2} \quad \text { at } A_{\beta \gamma} \\
& \text { BC4 } \quad \mu_{\beta} \mathbf{n}_{\beta \gamma} \cdot\left[-\mathbf{I} \mathbf{a}_{\beta \gamma 2}+\left(\nabla \mathbf{A}_{\beta \gamma 2}+\nabla \mathbf{A}_{\beta \gamma 2}^{T}\right)\right] \\
& =\mu_{\gamma} \mathbf{n}_{\beta \gamma} \cdot\left[-\mathbf{I} \mathbf{a}_{\gamma \gamma 2}+\left(\nabla \mathbf{A}_{\gamma \gamma 2}+\nabla \mathbf{A}_{\gamma \gamma 2}^{T}\right)\right] \text { at } A_{\beta \gamma}
\end{aligned}
$$

Periodicity

$$
\begin{aligned}
\mathbf{A}_{\alpha \gamma 2}\left(\mathbf{r}+\mathbf{l}_{i}\right) & =\mathbf{A}_{\alpha \gamma 2}(\mathbf{r}) \quad \alpha=\beta \text { and } \gamma \quad i=1,2,3 \\
\mathbf{a}_{\alpha \gamma 2}\left(\mathbf{r}+\mathbf{l}_{i}\right) & =\mathbf{a}_{\alpha \gamma 2}(\mathbf{r}) \quad \alpha=\beta \text { and } \gamma \quad i=1,2,3
\end{aligned}
$$

Average

$$
\left\langle\mathbf{A}_{\alpha \gamma 2}\right\rangle^{\alpha}=0 \quad \alpha=\beta \text { and } \gamma
$$

In writing the momentum equations (82a) and (83a), we have used the definitions of the inertial correction tensor for each phase $\mathbf{F}_{\alpha \alpha}(\alpha=\beta, \gamma)$ and the coupling inertial correction tensors $\mathbf{F}_{\gamma \beta}$ and $\mathbf{F}_{\beta \gamma}$ given by

$$
\frac{1}{V_{\alpha}} \int_{A_{\alpha \sigma} \cup A_{\beta \gamma}} \mathbf{n}_{\alpha} \cdot\left(-\mathbf{I a}_{\alpha \kappa 2}+\nabla \mathbf{A}_{\alpha \kappa 2}\right) \mathrm{d} A=-\varepsilon_{\kappa} \mathbf{K}_{\alpha}^{-1} \cdot \mathbf{F}_{\alpha \kappa} \quad \alpha, \kappa=\beta, \gamma
$$

It shall be noted that the solution of the microscopic flow problem is required prior to that of the four boundary value problems described above, allowing the determination of the eight permeability and inertial correction tensors. Since these tensors are computed from boundary value problems with periodic boundary conditions over a periodic unit cell, the microscopic flow must also be computed on the same unit cell using the steady version of 
Eqs. 2-7 where boundary conditions BC5 and BC6 in Eq. 7 are replaced by periodic ones. Moreover, the solution of this microscopic flow provides the location of the $A_{\beta \gamma}$ interface necessary to solve the four problems I1, II1, I2 and II2, noting that problems I1 and II1 are purely geometrical ones. On the other hand, it provides the microscopic velocity fields $\mathbf{v}_{\beta}$ and $\mathbf{v}_{\gamma}$ necessary to solve problems I2 and II 2 .

At this point, we are in position to move on to the macroscopic closed form of the inertial two-phase flow process under consideration.

\section{Macroscopic Equations}

Before introducing the representations of the deviations as functions of the average velocities in the average momentum equations, macroscopic acceleration and convective inertial terms in these last equations need to be reinspected. This is motivated by the time-scale constraint introduced in Eq. 55 as a requirement to simplify the closure problem. In addition, our interest is to examine the conditions under which macroscopic inertial terms are significant. To do so, we recall the average momentum equation in its last version of Eq. 37 as

$$
\begin{aligned}
& \rho_{\alpha} \frac{\partial\left\langle\mathbf{v}_{\alpha}\right\rangle^{\alpha}}{\partial t}+\rho_{\alpha}\left\langle\mathbf{v}_{\alpha}\right\rangle^{\alpha} \cdot \nabla\left\langle\mathbf{v}_{\alpha}\right\rangle^{\alpha}+\rho_{\alpha} \varepsilon_{\alpha}^{-1} \nabla \cdot\left\langle\tilde{\mathbf{v}}_{\alpha} \tilde{\mathbf{v}}_{\alpha}\right\rangle=-\nabla\left\langle p_{\alpha}\right\rangle^{\alpha}+\rho_{\alpha} \mathbf{g} \\
& +\frac{1}{V_{\alpha}} \int_{A_{\alpha \sigma} \cup A_{\beta \gamma}} \mathbf{n}_{\alpha} \cdot\left(-\mathbf{I} \tilde{p}_{\alpha}+\mu_{\alpha} \nabla \tilde{\mathbf{v}}_{\alpha}\right) \mathrm{d} A \quad \alpha=\beta, \gamma
\end{aligned}
$$

The order of magnitude of the two macroscopic inertial terms is the same and, on the basis of Eq. 33, is given by

$$
\begin{aligned}
& \rho_{\alpha}\left\langle\mathbf{v}_{\alpha}\right\rangle^{\alpha} \cdot \nabla\left\langle\mathbf{v}_{\alpha}\right\rangle^{\alpha}=O\left(\frac{\rho_{\alpha}\left\|\left\langle\mathbf{v}_{\alpha}\right\rangle^{\alpha}\right\|^{2}}{L}\right) \quad \alpha=\beta, \gamma \\
& \rho_{\alpha} \varepsilon_{\alpha}^{-1} \nabla \cdot\left\langle\tilde{\mathbf{v}}_{\alpha} \tilde{\mathbf{v}}_{\alpha}\right\rangle=O\left(\frac{\rho_{\alpha}\left\|\left\langle\mathbf{v}_{\alpha}\right\rangle^{\alpha}\right\|^{2}}{L}\right) \quad \alpha=\beta, \gamma
\end{aligned}
$$

Recalling Eq. 34, the order of magnitude of the interfacial viscous stress term is

$$
\frac{\mu_{\alpha}}{V_{\alpha}} \int_{A_{\alpha \sigma} \cup A_{\beta \gamma}} \mathbf{n}_{\alpha} \cdot \nabla \tilde{\mathbf{v}}_{\alpha} \mathrm{d} A=\mathbf{O}\left(\frac{\mu_{\alpha}\left\langle\mathbf{v}_{\alpha}\right\rangle^{\alpha}}{l_{\alpha}^{2}}\right) \quad \alpha=\beta, \gamma
$$

Clearly, the two macroscopic inertial terms are negligible in comparison to this last integral term in the averaged momentum equations when the following constraint is satisfied

$$
\max _{\alpha=\beta, \gamma}\left(\frac{\rho_{\alpha}\left\|\left\langle\mathbf{v}_{\alpha}\right\rangle^{\alpha}\right\| l_{\alpha}}{\mu_{\alpha}} \frac{l_{\alpha}}{L}\right) \ll 1
$$

From a practical point of view, a safe constraint would be

$$
\frac{l_{p}}{L} \max _{\alpha=\beta, \gamma}\left(\frac{R e_{\alpha}}{\varepsilon_{\alpha}}\right) \ll 1
$$


where $R e_{\alpha}$ is a Reynolds number based on the mean pore-size $l_{p}$, associated to the $\alpha$-phase and defined by

$$
\operatorname{Re}_{\alpha}=\frac{\rho_{\alpha}\left\|\left\langle\mathbf{v}_{\alpha}\right\rangle\right\| l_{p}}{\mu_{\alpha}} \quad \alpha=\beta, \gamma
$$

Under this constraint, the macroscopic momentum balance equation can be written as

$$
\begin{aligned}
\rho_{\alpha} \frac{\partial\left\langle\mathbf{v}_{\alpha}\right\rangle^{\alpha}}{\partial t}= & -\nabla\left\langle p_{\alpha}\right\rangle^{\alpha}+\rho_{\alpha} \mathbf{g} \\
& +\frac{1}{V_{\alpha}} \int_{A_{\alpha \sigma} \cup A_{\beta \gamma}} \mathbf{n}_{\alpha} \cdot\left(-\mathbf{I} \tilde{p}_{\alpha}+\mu_{\alpha} \nabla \tilde{\mathbf{v}}_{\alpha}\right) \mathrm{d} A \quad \alpha=\beta, \gamma
\end{aligned}
$$

We now turn our attention to the acceleration term and consider its order of magnitude which is given by

$$
\rho_{\alpha} \frac{\partial\left\langle\mathbf{v}_{\alpha}\right\rangle^{\alpha}}{\partial t}=\mathbf{O}\left(\frac{\rho_{\alpha}\left\langle\mathbf{v}_{\alpha}\right\rangle^{\alpha}}{t^{*}}\right) \quad \alpha=\beta, \gamma
$$

Under the constraint already indicated by Eq. 51, i.e.

$$
t^{*} \gg \max _{\alpha=\beta, \gamma}\left(\frac{\rho_{\alpha} l_{\alpha}^{2}}{\mu_{\alpha}}\right)
$$

it can be clearly seen that

$$
\rho_{\alpha} \frac{\partial\left\langle\mathbf{v}_{\alpha}\right\rangle^{\alpha}}{\partial t} \ll \frac{\mu_{\alpha}}{V_{\alpha}} \int_{A_{\alpha \sigma} \cup A_{\beta \gamma}} \mathbf{n}_{\alpha} \cdot \nabla \tilde{\mathbf{v}}_{\alpha} \mathrm{d} A \quad \alpha=\beta, \gamma
$$

so that the average momentum equation is finally

$$
0=-\nabla\left\langle p_{\alpha}\right\rangle^{\alpha}+\rho_{\alpha} \mathbf{g}+\frac{1}{V_{\alpha}} \int_{A_{\alpha \sigma} \cup A_{\beta \gamma}} \mathbf{n}_{\alpha} \cdot\left(-\mathbf{I} \tilde{p}_{\alpha}+\mu_{\alpha} \nabla \tilde{\mathbf{v}}_{\alpha}\right) \mathrm{d} A \quad \alpha=\beta, \gamma
$$

Here, it must be emphasized that this form of the macroscopic equation results from two important constraints: the time-scale constraint expressed by Eq. 51 on the one hand and the constraint given by (89) on the other hand. The former was discussed earlier in the development and indicates that the non-stationary (or time acceleration) term in the momentum balance is negligible both at the micro- and macroscopic scales. From the latter, we can notice that macroscopic inertial forces are negligible and are not the source of inertia at this scale. In fact, as will be seen later, macroscopic nonlinearity originates from microscopic inertia which is entirely contained in the convective term $\rho_{\alpha} \mathbf{v}_{\alpha} \cdot \nabla \tilde{\mathbf{v}}_{\alpha}$ of the closure problem in Eq. 71. This is a situation similar to that encountered for one-phase flow where exactly the same observation was made by Hassanizadeh and Gray (1987) and Whitaker (1996). 
We are now ready to obtain the closed form by introducing the representations of the deviations detailed above in this last equation. This yields (for the $\alpha$-phase)

$$
\begin{aligned}
0= & -\nabla\left\langle p_{\alpha}\right\rangle^{\alpha}+\rho_{\alpha} \mathbf{g} \\
& +\mu_{\alpha}\left\{\frac{1}{V_{\alpha}} \int_{A_{\alpha \sigma} \cup A_{\beta \gamma}} \mathbf{n}_{\alpha} \cdot\left(-\mathbf{I} \mathbf{a}_{\alpha \alpha 1}+\nabla \mathbf{A}_{\alpha \alpha 1}\right) \mathrm{d} A\right\} \cdot\left\langle\mathbf{v}_{\alpha}\right\rangle^{\alpha} \\
& +\mu_{\alpha}\left\{\frac{1}{V_{\alpha}} \int_{A_{\alpha \sigma} \cup A_{\beta \gamma}} \mathbf{n}_{\alpha} \cdot\left(-\mathbf{I a}_{\alpha \alpha 2}+\nabla \mathbf{A}_{\alpha \alpha 2}\right) \mathrm{d} A\right\} \cdot\left\langle\mathbf{v}_{\alpha}\right\rangle^{\alpha} \\
& \left.+\mu_{\alpha}\left\{\frac{1}{V_{\alpha}} \int_{A_{\alpha \sigma} \cup A_{\beta \gamma}} \mathbf{n}_{\alpha} \cdot\left(-\mathbf{I} \mathbf{a}_{\alpha \kappa 1}+\nabla \mathbf{A}_{\alpha \kappa 1}\right) \mathrm{d} A\right\} \cdot\left\langle\mathbf{v}_{\kappa}\right\rangle^{\kappa}\right] \cdot\left\langle\mathbf{v}_{\kappa}\right\rangle^{\kappa} \quad \alpha, \kappa=\beta, \gamma, \quad \alpha \neq \kappa \\
& +\mu_{\alpha}\left\{\frac{1}{V_{\alpha}} \int_{A_{\alpha \sigma} \cup A_{\beta \gamma}} \mathbf{n}_{\alpha} \cdot\left(-\mathbf{I} \mathbf{a}_{\alpha \kappa 2}+\nabla \mathbf{A}_{\alpha \kappa 2}\right) \mathrm{d} A\right\}
\end{aligned}
$$

where all the area integrals can be identified from the definitions of the permeability, viscous drag and inertial correction tensors. Using the relationships (80), (81) and (84), we can write

$$
\begin{aligned}
0= & -\nabla\left\langle p_{\alpha}\right\rangle^{\alpha}+\rho_{\alpha} \mathbf{g}-\mu_{\alpha} \mathbf{K}_{\alpha}^{-1} \cdot\left\langle\mathbf{v}_{\alpha}\right\rangle-\mu_{\alpha} \mathbf{K}_{\alpha}^{-1} \cdot \mathbf{F}_{\alpha \alpha} \cdot\left\langle\mathbf{v}_{\alpha}\right\rangle \\
& +\mu_{\alpha} \mathbf{K}_{\alpha}^{-1} \cdot \mathbf{K}_{\alpha \kappa} \cdot\left\langle\mathbf{v}_{\kappa}\right\rangle-\mu_{\alpha} \mathbf{K}_{\alpha}^{-1} \cdot \mathbf{F}_{\alpha \kappa} \cdot\left\langle\mathbf{v}_{\kappa}\right\rangle \quad \alpha, \kappa=\beta, \gamma, \quad \alpha \neq \kappa
\end{aligned}
$$

Rearranging, this gives

$$
\begin{aligned}
\left\langle\mathbf{v}_{\alpha}\right\rangle= & -\frac{\mathbf{K}_{\alpha}}{\mu_{\alpha}} \cdot\left(\nabla\left\langle p_{\alpha}\right\rangle^{\alpha}-\rho_{\alpha} \mathbf{g}\right)-\mathbf{F}_{\alpha \alpha} \cdot\left\langle\mathbf{v}_{\alpha}\right\rangle \\
& +\mathbf{K}_{\alpha \kappa} \cdot\left\langle\mathbf{v}_{\kappa}\right\rangle-\mathbf{F}_{\alpha \kappa} \cdot\left\langle\mathbf{v}_{\kappa}\right\rangle \quad \alpha, \kappa=\beta, \gamma, \quad \alpha \neq \kappa
\end{aligned}
$$

which represents our final macroscopic momentum balance equation for two-phase inertial flow in homogeneous porous media under all the constraints detailed in the course of the development. As will be shown later, it must be emphasized that $\mathbf{K}_{\alpha}$ and $\mathbf{K}_{\alpha \kappa}$ are intrinsic tensors while $\mathbf{F}_{\alpha \alpha}$ and $\mathbf{F}_{\alpha \kappa}$ are velocity dependent. If inertial effects are absent, these equations have the same form as those obtained for two-phase creeping flow in homogeneous porous media, i.e. (Whitaker 1994)

$$
\left\langle\mathbf{v}_{\alpha}\right\rangle=-\frac{\mathbf{K}_{\alpha}}{\mu_{\alpha}} \cdot\left(\nabla\left\langle p_{\alpha}\right\rangle^{\alpha}-\rho_{\alpha} \mathbf{g}\right)+\mathbf{K}_{\alpha \kappa} \cdot\left\langle\mathbf{v}_{\kappa}\right\rangle \quad \alpha, \kappa=\beta, \gamma, \quad \alpha \neq \kappa
$$

The importance of the viscous coupling present in such a model has been the subject of active work (Kalaydjian 1990; Rose 1989; Zarcone 1994) and an order of magnitude was developed by Whitaker (1994) leading to $\mathbf{K}_{\beta \gamma} \cdot \mathbf{K}_{\gamma \beta}=\mathbf{O}(\mathbf{I})$. Later, Lasseux et al. (1996) derived an exact relationship between these two tensors given by

$$
\mu_{\alpha} \mathbf{K}_{\alpha \kappa} \cdot \mathbf{K}_{\kappa}=\mu_{\kappa} \mathbf{K}_{\alpha} \cdot \mathbf{K}_{\kappa \alpha}^{T} \quad \alpha, \kappa=\beta, \gamma, \quad \alpha \neq \kappa
$$

In the same reference, it was shown that a more convenient form than that of Eq. 100 is

$$
\left\langle\mathbf{v}_{\alpha}\right\rangle=-\frac{\mathbf{K}_{\alpha \alpha}^{*}}{\mu_{\alpha}} \cdot\left(\nabla\left\langle p_{\alpha}\right\rangle^{\alpha}-\rho_{\alpha} \mathbf{g}\right)-\mathbf{K}_{\alpha \kappa}^{*} \cdot\left(\nabla\left\langle p_{\kappa}\right\rangle^{\kappa}-\rho_{\kappa} \mathbf{g}\right) \quad \alpha, \kappa=\beta, \gamma, \quad \alpha \neq \kappa
$$


in accordance with a result obtained by the homogenization technique (Auriault 1987). The dominant and coupling permeability tensors $\mathbf{K}_{\alpha \alpha}^{*}$ and $\mathbf{K}_{\alpha \kappa}^{*}(\alpha, \kappa=\beta, \gamma ; \alpha \neq \kappa)$ appearing in this last equation are related to the tensors of Eq. 100 by

$$
\begin{aligned}
& \mathbf{K}_{\alpha \alpha}^{*}=\left(\mathbf{I}-\mathbf{K}_{\alpha \kappa} \cdot \mathbf{K}_{\kappa \alpha}\right)^{-1} \cdot \mathbf{K}_{\alpha} \quad \alpha, \kappa=\beta, \gamma \quad \alpha \neq \kappa \\
& \mathbf{K}_{\alpha \kappa}^{*}=\left(\mathbf{I}-\mathbf{K}_{\alpha \kappa} \cdot \mathbf{K}_{\kappa \alpha}\right)^{-1} \cdot\left(\mathbf{K}_{\alpha \kappa} \cdot \mathbf{K}_{\kappa}\right) \quad \alpha, \kappa=\beta, \gamma \quad \alpha \neq \kappa
\end{aligned}
$$

and are given by closure problems that are recalled in Appendix 2 of this article. These closure problems are rearranged versions of problems I1 and II1 given by Eqs. 78 and 79 . Following the same lines, we shall prefer the following macroscopic momentum equation in the case under study in the present work

$$
\begin{aligned}
\left\langle\mathbf{v}_{\alpha}\right\rangle= & -\frac{\mathbf{K}_{\alpha \alpha}^{*}}{\mu_{\alpha}} \cdot\left(\nabla\left\langle p_{\alpha}\right\rangle^{\alpha}-\rho_{\alpha} \mathbf{g}\right)-\mathbf{F}_{\alpha \alpha} \cdot\left\langle\mathbf{v}_{\alpha}\right\rangle \\
& -\frac{\mathbf{K}_{\alpha \kappa}^{*}}{\mu_{\kappa}} \cdot\left(\nabla\left\langle p_{\kappa}\right\rangle^{\kappa}-\rho_{\kappa} \mathbf{g}\right)-\mathbf{F}_{\alpha \kappa} \cdot\left\langle\mathbf{v}_{\kappa}\right\rangle \quad \alpha, \kappa=\beta, \gamma, \quad \alpha \neq \kappa
\end{aligned}
$$

In the next section, we provide alternate versions of the closure problems the solutions of which are required to find the inertial correction tensors.

\section{Procedure for Obtaining the F Tensors}

In this section, we present a more tractable form of the boundary value problems that are to be solved to determine the four inertial tensors $\mathbf{F}_{\beta \beta}, \mathbf{F}_{\gamma \gamma}, \mathbf{F}_{\beta \gamma}$ and $\mathbf{F}_{\gamma \beta}$. Rather than starting from the closure problems I 2 and II 2 given by Eqs. 82 and 83, it is more convenient to start from the forms I and II of Eqs. 74 and 75 and we recall them as

Problem I

$$
\begin{aligned}
& \left(\rho_{\alpha} \mathbf{v}_{\alpha} / \mu_{\alpha}\right) \cdot \nabla \mathbf{A}_{\alpha \beta}=-\nabla \mathbf{a}_{\alpha \beta}+\nabla^{2} \mathbf{A}_{\alpha \beta} \\
& \quad+\varepsilon_{\beta}\left[\delta_{\alpha \beta} \mathbf{H}_{\beta}^{-1}+\left(\delta_{\alpha \beta}-1\right) \mathbf{K}_{\gamma}^{-1} \cdot \mathbf{H}_{\gamma \beta}\right] \quad \text { in } V_{\alpha}, \quad \alpha=\beta \text { and } \gamma \\
& \nabla \cdot \mathbf{A}_{\alpha \beta}=0 \quad \text { in } V_{\alpha}, \quad \alpha=\beta \text { and } \gamma \\
& \text { BC1 and BC2 } \quad \mathbf{A}_{\alpha \beta}=-\delta_{\alpha \beta} \mathbf{I} \quad \text { at } A_{\alpha \sigma}, \quad \alpha=\beta \text { and } \gamma \\
& \text { BC3 } \quad \mathbf{A}_{\beta \beta}=\mathbf{A}_{\gamma \beta}-\mathbf{I} \quad \text { at } A_{\beta \gamma} \\
& \text { BC4 } \quad \mu_{\beta} \mathbf{n}_{\beta \gamma} \cdot\left[-\mathbf{I} \mathbf{a}_{\beta \beta}+\left(\nabla \mathbf{A}_{\beta \beta}+\nabla \mathbf{A}_{\beta \beta}^{T}\right)\right] \\
& =\mu_{\gamma} \mathbf{n}_{\beta \gamma} \cdot\left[-\mathbf{I} \mathbf{a}_{\gamma \beta}+\left(\nabla \mathbf{A}_{\gamma \beta}+\nabla \mathbf{A}_{\gamma \beta}^{T}\right)\right] \text { at } A_{\beta \gamma}
\end{aligned}
$$

Periodicity

$$
\begin{aligned}
& \mathbf{a}_{\alpha \beta}\left(\mathbf{r}+\mathbf{l}_{i}\right)=\mathbf{a}_{\alpha \beta}(\mathbf{r}) \quad \alpha=\beta \text { and } \gamma \quad i=1,2,3 \\
& \mathbf{A}_{\alpha \beta}\left(\mathbf{r}+\mathbf{l}_{i}\right)=\mathbf{A}_{\alpha \beta}(\mathbf{r}) \quad \alpha=\beta \text { and } \gamma \quad i=1,2,3
\end{aligned}
$$

Average

$$
\left\langle\mathbf{A}_{\alpha \beta}\right\rangle^{\alpha}=0 \quad \alpha=\beta \text { and } \gamma
$$


Problem II

$$
\begin{aligned}
& \left(\rho_{\alpha} \mathbf{v}_{\alpha} / \mu_{\alpha}\right) \cdot \nabla \mathbf{A}_{\alpha \gamma}=-\nabla \mathbf{a}_{\alpha \gamma}+\nabla^{2} \mathbf{A}_{\alpha \gamma} \\
& \quad+\varepsilon_{\gamma}\left[\delta_{\alpha \gamma} \mathbf{H}_{\gamma}^{-1}+\left(\delta_{\alpha \gamma}-1\right) \mathbf{K}_{\beta}^{-1} \cdot \mathbf{H}_{\beta \gamma}\right] \quad \text { in } V_{\alpha}, \quad \alpha=\beta \text { and } \gamma \\
& \nabla \cdot \mathbf{A}_{\alpha \gamma}=0 \quad \text { in } V_{\alpha}, \quad \alpha=\beta \text { and } \gamma \\
& \text { BC1 and BC2 } \quad \mathbf{A}_{\alpha \gamma}=-\delta_{\alpha \gamma} \mathbf{I} \text { at } A_{\alpha \sigma}, \quad \alpha=\beta \text { and } \gamma \\
& \text { BC3 } \quad \mathbf{A}_{\beta \gamma}=\mathbf{A}_{\gamma \gamma}+\mathbf{I} \quad \text { at } A_{\beta \gamma} \\
& \text { BC4 } \quad \mu_{\beta} \mathbf{n}_{\beta \gamma} \cdot\left[-\mathbf{I a}_{\beta \gamma}+\left(\nabla \mathbf{A}_{\beta \gamma}+\nabla \mathbf{A}_{\beta \gamma}^{T}\right)\right] \\
& =\mu_{\gamma} \mathbf{n}_{\beta \gamma} \cdot\left[-\mathbf{I} \mathbf{a}_{\gamma \gamma}+\left(\nabla \mathbf{A}_{\gamma \gamma}+\nabla \mathbf{A}_{\gamma \gamma}^{T}\right)\right] \text { at } A_{\beta \gamma}
\end{aligned}
$$

Periodicity

$$
\begin{aligned}
& \mathbf{a}_{\alpha \gamma}\left(\mathbf{r}+\mathbf{l}_{i}\right)=\mathbf{a}_{\alpha \gamma}(\mathbf{r}) \quad \alpha=\beta \text { and } \gamma \quad i=1,2,3 \\
& \mathbf{A}_{\alpha \gamma}\left(\mathbf{r}+\mathbf{l}_{i}\right)=\mathbf{A}_{\alpha \gamma}(\mathbf{r}) \quad \alpha=\beta \text { and } \gamma \quad i=1,2,3
\end{aligned}
$$

Average

$$
\left\langle\mathbf{A}_{\alpha \gamma}\right\rangle^{\alpha}=0 \quad \alpha=\beta \text { and } \gamma
$$

Here, we have used the tensors $\mathbf{H}_{\beta}, \mathbf{H}_{\gamma \beta}, \mathbf{H}_{\gamma}$, and $\mathbf{H}_{\beta \gamma}$ defined by

$$
\begin{gathered}
\varepsilon_{\alpha} \mathbf{H}_{\alpha}^{-1}=-\frac{1}{V_{\alpha}} \int_{A_{\alpha \sigma} \cup A_{\beta \gamma}} \mathbf{n}_{\alpha} \cdot\left(-\mathbf{I} \mathbf{a}_{\alpha \alpha}+\nabla \mathbf{A}_{\alpha \alpha}\right) \mathrm{d} A \quad \alpha=\beta, \gamma \\
\varepsilon_{\kappa} \mathbf{K}_{\alpha}^{-1} \cdot \mathbf{H}_{\alpha \kappa}=\frac{1}{V_{\alpha}} \int_{A_{\alpha \sigma} \cup A_{\beta \gamma}} \mathbf{n}_{\alpha} \cdot\left(-\mathbf{I a}_{\alpha \kappa}+\nabla \mathbf{A}_{\alpha \kappa}\right) \mathrm{d} A \quad \alpha, \kappa=\beta, \gamma, \quad \alpha \neq \kappa
\end{gathered}
$$

and it shall be noted that

$$
\begin{gathered}
\mathbf{H}_{\alpha}^{-1}=\mathbf{K}_{\alpha}^{-1} \cdot\left(\mathbf{I}+\mathbf{F}_{\alpha \alpha}\right) \quad \alpha=\beta, \gamma \\
\mathbf{H}_{\alpha \kappa}=\mathbf{K}_{\alpha \kappa}-\mathbf{F}_{\alpha \kappa} \quad \alpha, \kappa=\beta, \gamma, \quad \alpha \neq \kappa
\end{gathered}
$$

Under their initial forms, these two problems are integro-differential ones and simpler versions are desirable for tractable resolution. This can be performed by setting

$$
\begin{aligned}
\mathbf{A}_{\alpha \kappa}= & -\delta_{\alpha \kappa} \mathbf{I} \\
& -\varepsilon_{\kappa}\left[\mathbf{A}_{\alpha \kappa}^{\mathbf{0}} \cdot \mathbf{H}_{\kappa}^{-1}-\mathbf{A}_{\alpha \xi}^{\mathbf{0}} \cdot\left(\mathbf{K}_{\xi}^{-1} \cdot \mathbf{H}_{\xi \kappa}\right)\right] \quad \alpha, \kappa, \xi=\beta, \gamma, \quad \xi \neq \kappa
\end{aligned}
$$

and

$$
\mathbf{a}_{\alpha \kappa}=-\varepsilon_{\kappa}\left[\mathbf{a}_{\alpha \kappa}^{\mathbf{0}} \cdot \mathbf{H}_{\kappa}^{-1}-\mathbf{a}_{\alpha \xi}^{\mathbf{0}} \cdot\left(\mathbf{K}_{\xi}^{-1} \cdot \mathbf{H}_{\xi \kappa}\right)\right] \quad \alpha, \kappa, \xi=\beta, \gamma, \quad \xi \neq \kappa
$$

When these decompositions are replaced in the above problems I and II, and because of the absence of the curvature in the stress jump boundary condition at $A_{\beta \gamma}$, the two following forms of the closure problems can be used to compute the inertial correction tensors 
Problem I(a)

$$
\begin{aligned}
& \left(\rho_{\alpha} \mathbf{v}_{\alpha} / \mu_{\alpha}\right) \cdot \nabla \mathbf{A}_{\alpha \beta}^{0}=-\nabla \mathbf{a}_{\alpha \beta}^{0}+\nabla^{2} \mathbf{A}_{\alpha \beta}^{0}-\delta_{\alpha \beta} \mathbf{I} \quad \text { in } V_{\alpha}, \quad \alpha=\beta \text { and } \gamma \\
& \nabla \cdot \mathbf{A}_{\alpha \beta}^{0}=0 \quad \text { in } V_{\alpha}, \quad \alpha=\beta \text { and } \gamma \\
& \mathrm{BC} 1 \text { and BC2 } \quad \mathbf{A}_{\alpha \beta}^{0}=0 \quad \text { at } A_{\alpha \sigma}, \quad \alpha=\beta \text { and } \gamma \\
& \mathrm{BC} 3 \quad \mathbf{A}_{\beta \beta}^{0}=\mathbf{A}_{\gamma \beta}^{0} \quad \text { at } A_{\beta \gamma} \\
& \mathrm{BC} 4 \quad \mu_{\beta} \mathbf{n}_{\beta \gamma} \cdot\left[-\mathbf{I a}_{\beta \beta}^{0}+\left(\nabla \mathbf{A}_{\beta \beta}^{0}+\nabla \mathbf{A}_{\beta \beta}^{0 T}\right)\right] \\
& =\mu_{\gamma} \mathbf{n}_{\beta \gamma} \cdot\left[-\mathbf{I} \mathbf{a}_{\gamma \beta}^{0}+\left(\nabla \mathbf{A}_{\gamma \beta}^{0}+\nabla \mathbf{A}_{\gamma \beta}^{0 T}\right)\right] \text { at } A_{\beta \gamma}
\end{aligned}
$$

Periodicity

$$
\begin{aligned}
& \mathbf{a}_{\alpha \beta}^{0}\left(\mathbf{r}+\mathbf{l}_{i}\right)=\mathbf{a}_{\alpha \beta}^{0}(\mathbf{r}) \quad \alpha=\beta \text { and } \gamma \quad i=1,2,3 \\
& \mathbf{A}_{\alpha \beta}^{0}\left(\mathbf{r}+\mathbf{l}_{i}\right)=\mathbf{A}_{\alpha \beta}^{0}(\mathbf{r}) \quad \alpha=\beta \text { and } \gamma \quad i=1,2,3
\end{aligned}
$$

Problem II(a)

$$
\begin{aligned}
& \left(\rho_{\alpha} \mathbf{v}_{\alpha} / \mu_{\alpha}\right) \cdot \nabla \mathbf{A}_{\alpha \gamma}^{0}=-\nabla \mathbf{a}_{\alpha \gamma}^{0}+\nabla^{2} \mathbf{A}_{\alpha \gamma}^{0}-\delta_{\alpha \gamma} \mathbf{I} \quad \text { in } V_{\alpha}, \quad \alpha=\beta \text { and } \gamma \\
& \nabla \cdot \mathbf{A}_{\alpha \gamma}^{0}=0 \quad \text { in } V_{\alpha}, \quad \alpha=\beta \text { and } \gamma \\
& \mathrm{BC} 1 \text { and BC2 } \quad \mathbf{A}_{\alpha \gamma}^{0}=0 \quad \text { at } A_{\alpha \sigma}, \quad \alpha=\beta \text { and } \gamma \\
& \mathrm{BC} 3 \quad \mathbf{A}_{\beta \gamma}^{0}=\mathbf{A}_{\gamma \gamma}^{0} \quad \text { at } A_{\beta \gamma} \\
& \mathrm{BC} 4 \quad \mu_{\beta} \mathbf{n}_{\beta \gamma} \cdot\left[-\mathbf{I a}_{\beta \gamma}^{0}+\left(\nabla \mathbf{A}_{\beta \gamma}^{0}+\nabla \mathbf{A}_{\beta \gamma}^{0 T}\right)\right] \\
& =\mu_{\gamma} \mathbf{n}_{\beta \gamma} \cdot\left[-\mathbf{I} \mathbf{a}_{\gamma \gamma}^{0}+\left(\nabla \mathbf{A}_{\gamma \gamma}^{0}+\nabla \mathbf{A}_{\gamma \gamma}^{0 T}\right)\right] \text { at } A_{\beta \gamma}
\end{aligned}
$$

Periodicity

$$
\begin{aligned}
& \mathbf{a}_{\alpha \gamma}^{0}\left(\mathbf{r}+\mathbf{l}_{i}\right)=\mathbf{a}_{\alpha \gamma}^{0}(\mathbf{r}) \quad \alpha=\beta \text { and } \gamma \quad i=1,2,3 \\
& \mathbf{A}_{\alpha \gamma}^{0}\left(\mathbf{r}+\mathbf{l}_{i}\right)=\mathbf{A}_{\alpha \gamma}^{0}(\mathbf{r}) \quad \alpha=\beta \text { and } \gamma \quad i=1,2,3
\end{aligned}
$$

Averages

$$
\begin{aligned}
\mathbf{H}_{\alpha} & =\varepsilon_{\alpha}\left(\left\langle\mathbf{A}_{\alpha \kappa}^{0}\right\rangle^{\alpha} \cdot\left\langle\mathbf{A}_{\kappa \kappa}^{0}\right\rangle^{\kappa^{-1}} \cdot\left\langle\mathbf{A}_{\kappa \alpha}^{0}\right\rangle^{\kappa}-\left\langle\mathbf{A}_{\alpha \alpha}^{0}\right\rangle^{\alpha}\right) \quad \alpha, \kappa=\beta, \gamma, \quad \alpha \neq \kappa \\
\mathbf{H}_{\alpha \kappa} & =\mathbf{K}_{\alpha} \cdot\left\langle\mathbf{A}_{\alpha \alpha}^{0}\right\rangle^{\alpha^{-1}} \cdot\left\langle\mathbf{A}_{\alpha \kappa}^{0}\right\rangle^{\alpha} \cdot \mathbf{H}_{\kappa}^{-1} \quad \alpha, \kappa=\beta, \gamma, \quad \alpha \neq \kappa
\end{aligned}
$$

Each of these two problems has basically a structure equivalent to that of an incompressible Navier-Stokes two-phase flow without any dependence on the curvature of the fluid-fluid interface. When a solver is available to compute the initial microscopic physical flow problem, the solution of which includes the location of the interface as well as the determination of $\mathbf{v}_{\beta}$ and $\mathbf{v}_{\gamma}$, the same solver can be used to solve problems I(a) and II(a) of Eqs. 114 and 115 and determine the four tensors $\mathbf{H}_{\beta}, \mathbf{H}_{\gamma \beta}, \mathbf{H}_{\gamma}$ and $\mathbf{H}_{\beta \gamma}$. Note that the computation of these four tensors requires $\mathbf{K}_{\beta}$ and $\mathbf{K}_{\gamma}$ that can be obtained according to the procedure provided in Appendix 2 of this article. Finally, the inertial correction tensors are obtained by making use of the relations (110) and (111), i.e.

$$
\begin{aligned}
& \mathbf{F}_{\alpha \alpha}=\mathbf{K}_{\alpha} \cdot \mathbf{H}_{\alpha}^{-1}-\mathbf{I} \quad \alpha=\beta, \gamma \\
& \mathbf{F}_{\alpha \kappa}=\mathbf{K}_{\alpha \kappa}-\mathbf{H}_{\alpha \kappa} \quad \alpha, \kappa=\beta, \gamma, \quad \alpha \neq \kappa
\end{aligned}
$$


In these last relationships, $\mathbf{K}_{\gamma \beta}$ and $\mathbf{K}_{\beta \gamma}$ are the two viscous drag tensors obtained from the solution of the boundary value problems also given in Appendix B.

\section{Conclusions}

In this work, we have derived the macroscopic model for some inertial two-phase, incompressible, Newtonian fluid flow through homogenous porous media. The validity of this model is subject to constraints on the length-scales and time-scales as well as on quantities involving the capillary, Reynolds and Weber numbers and assumes that fluctuations of the curvature of the fluid-fluid interface are unimportant over the unit cell representing the porous medium. The averaged continuity equation takes the same form as that obtained when inertia is negligible. The averaged momentum balance equations include generalized Darcy terms with viscous coupling as well as inertial and coupling inertial terms, each of these two last terms involving an inertial correction tensor. The two pairs of boundary value problems yielding the permeability and inertial correction tensors have been provided in simple enough versions for their solutions to be tractable using a two-phase Navier-Stokes solver. Numerical results are necessary to (i) identify the domain of validity of the macroscopic model through verification of the constraints for any special configuration in particular that corresponding to negligible capillary effects in the closure problem, (ii) estimate each of the inertial correction terms and in particular the contribution of the coupling inertial correction term.

Acknowledgements We wish to warmly thank Prof. Stephen Whitaker for fruitful discussions as well as Christian Moyne for his careful reading of the original manuscript.

\section{Appendix A}

The objective of this appendix is to derive a special form of the general transport theorem (Truesdell and Toupin 1960)

$$
\frac{\mathrm{d}}{\mathrm{d} t} \int_{\mathcal{V}(t)} \psi_{\alpha} \mathrm{d} V=\int_{\mathcal{V}(t)} \frac{\partial \psi_{\alpha}}{\partial t} \mathrm{~d} V+\int_{A(t)} \psi_{\alpha} \mathbf{n} \cdot \mathbf{w} \mathrm{d} A
$$

that can be used in the case of immiscible two-phase flow $(\alpha=\beta$ and $\gamma$ ) in a rigid porous medium. Even though this form is widely used in the literature, no detailed proof has been provided for this particular case.

In Eq. A1, $A(t)$ is the surface enclosing $\mathcal{V}(t), \mathbf{w}$ the velocity of a material point attached to $A(t)$ and $\mathbf{n}$ the unit vector normal to $A(t)$ pointing outside $\mathcal{V}(t)$. Without introducing any particularity, our result is derived for the $\beta$-phase by considering a quantity $\psi_{\beta}$ defined in $V_{\beta}(t)$ included at time $t$ in the arbitrary averaging volume $V$.

As depicted in Fig. 2, the volume $V_{\beta}(t)$ is enclosed at $t$ by the material surfaces $A_{\beta \sigma}(t)$ and $A_{\beta \gamma}(t)$ and by $A_{\beta V}(t)$, the latter representing the portion of the surface enclosing the averaging volume $V$ included in $V_{\beta}(t)$. Applying the theorem of Eq. A1 on $\psi_{\beta}$ with $\mathcal{V}(t)=V_{\beta}(t)$ yields

$$
\frac{\mathrm{d}}{\mathrm{d} t} \int_{\mathcal{V}_{\beta}(t)} \psi_{\beta} \mathrm{d} V=\int_{\mathcal{V}_{\beta}(t)} \frac{\partial \psi_{\beta}}{\partial t} \mathrm{~d} V+\int_{A_{\beta \sigma}(t) \cup A_{\beta \gamma}(t) \cup A_{\beta V}(t)} \psi_{\beta} \mathbf{n}_{\beta} \cdot \mathbf{w} \mathrm{d} A
$$




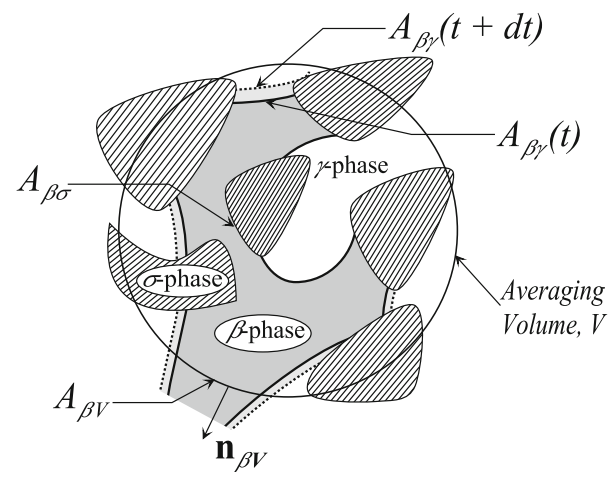

Fig. 2 The averaging volume $V$ containing the evolving volume $V_{\beta}(t)$ enclosed at $t$ by the surfaces $A_{\beta \sigma}(t)$, $A_{\beta \gamma}(t)$ and $A_{\beta V}(t)$

where $\mathbf{n}_{\beta}$ represents either $\mathbf{n}_{\beta \sigma}, \mathbf{n}_{\beta \gamma}$ or $\mathbf{n}_{\beta \mathbf{v}}$. As $A_{\beta \sigma}(t)$ and $A_{\beta \gamma}(t)$ are material surfaces and since no phase-change occurs on these surfaces, $\mathbf{w}=\mathbf{v}_{\beta}$ on $A_{\beta \sigma}(t)$ and $A_{\beta \gamma}(t)$. Moreover, due to the no-slip boundary condition on $A_{\beta \sigma}(t)$, the theorem takes the form

$$
\frac{\mathrm{d}}{\mathrm{d} t} \int_{\mathcal{V}_{\beta}(t)} \psi_{\beta} \mathrm{d} V=\int_{\mathcal{V}_{\beta}(t)} \frac{\partial \psi_{\beta}}{\partial t} \mathrm{~d} V+\int_{A_{\beta \gamma}(t)} \psi_{\beta} \mathbf{n}_{\beta} \cdot \mathbf{v}_{\beta} \mathrm{d} A+\int_{A_{\beta V}(t)} \psi_{\beta} \mathbf{n}_{\beta} \cdot \mathbf{w} \mathrm{d} A
$$

In addition, since $A_{\beta V}$ is part of the boundary of $V$ which is fixed in time, the velocity $\mathbf{w}$ is zero on this surface (see Fig. 2) leading to

$$
\frac{\mathrm{d}}{\mathrm{d} t} \int_{\mathcal{V}_{\beta}(t)} \psi_{\beta} \mathrm{d} V=\int_{\mathcal{V}_{\beta}(t)} \frac{\partial \psi_{\beta}}{\partial t} \mathrm{~d} V+\int_{A_{\beta \gamma}(t)} \psi_{\beta} \mathbf{n}_{\beta} \cdot \mathbf{v}_{\beta} \mathrm{d} A
$$

When this last form is divided by $V$, a fixed volume in time, one finally gets the special form of the transport theorem in the case of two-phase immiscible flow in a rigid porous medium, which for the $\beta$-phase is given by

$$
\frac{\mathrm{d}\left\langle\psi_{\beta}\right\rangle}{\mathrm{d} t}=\left\langle\frac{\partial \psi_{\beta}}{\partial t}\right\rangle+\frac{1}{V} \int_{A_{\beta \gamma}(t)} \psi_{\beta} \mathbf{n}_{\beta} \cdot \mathbf{v}_{\beta} \mathrm{d} A
$$

Since the average $\left\langle\psi_{\beta}\right\rangle$ is associated with the centroid of the averaging volume which is fixed in time, we can replace $\frac{\mathrm{d}\left\langle\psi_{\beta}\right\rangle}{\mathrm{d} t}$ by $\frac{\partial\left\langle\psi_{\beta}\right\rangle}{\partial t}$. Generalizing to the $\alpha$-phase $(\alpha=\beta$ or $\gamma)$ and using $\mathbf{v}_{\alpha}$, one has

$$
\left\langle\frac{\partial \mathbf{v}_{\alpha}}{\partial t}\right\rangle=\frac{\partial\left\langle\mathbf{v}_{\alpha}\right\rangle}{\partial t}-\frac{1}{V} \int_{A_{\beta \gamma}(t)} \mathbf{n}_{\alpha} \cdot \mathbf{v}_{\alpha} \mathbf{v}_{\alpha} \mathrm{d} A
$$

which can be used to average the acceleration term in the left-hand side of the original momentum Eq. 2 of the article to arrive at Eq. 18.

\section{Appendix B}

In this appendix, we simply list the result on the boundary value problems that must be solved in order to determine the four dominant and coupling permeability tensors $\mathbf{K}_{\alpha \alpha}^{*}$ and $\mathbf{K}_{\alpha \kappa}^{*}(\alpha$, 
$\kappa=\beta, \gamma, \alpha \neq \kappa)$ and we refer the reader to Lasseux et al. (1996) for the details. When the same nomenclature as that employed in this last reference is used, these problems are given by

Problem A-I

$$
\begin{aligned}
& -\nabla \mathbf{d}_{\alpha \beta}^{0}+\nabla^{2} \mathbf{D}_{\alpha \beta}^{0}=\delta_{\alpha \beta} \mathbf{I} \quad \text { in } V_{\alpha}, \quad \alpha=\beta \text { and } \gamma \\
& \nabla \cdot \mathbf{D}_{\alpha \beta}^{0}=0 \quad \text { in } V_{\alpha}, \quad \alpha=\beta \text { and } \gamma \\
& \mathrm{BC} 1 \text { and BC2 } \quad \mathbf{D}_{\alpha \beta}^{0}=0 \quad \text { at } A_{\alpha \sigma}, \quad \alpha=\beta \text { and } \gamma \\
& \mathrm{BC} 3 \quad \mathbf{D}_{\beta \beta}^{0}=\mathbf{D}_{\gamma \beta}^{0} \quad \text { at } A_{\beta \gamma} \\
& \mathrm{BC} 4 \quad \mu_{\beta} \mathbf{n}_{\beta \gamma} \cdot\left[-\mathbf{I} \mathbf{d}_{\beta \beta}^{0}+\left(\nabla \mathbf{D}_{\beta \beta}^{0}+\nabla \mathbf{D}_{\beta \beta}^{0 T}\right)\right] \\
& =\mu_{\gamma} \mathbf{n}_{\beta \gamma} \cdot\left[-\mathbf{I} \mathbf{d}_{\gamma \beta}^{0}+\left(\nabla \mathbf{D}_{\gamma \beta}^{0}+\nabla \mathbf{D}_{\gamma \beta}^{0 T}\right)\right] \text { at } A_{\beta \gamma}
\end{aligned}
$$

Periodicity:

$$
\begin{aligned}
& \mathbf{d}_{\alpha \beta}^{0}\left(\mathbf{r}+\mathbf{l}_{i}\right)=\mathbf{d}_{\alpha \beta}^{0}(\mathbf{r}) \quad \alpha=\beta \text { and } \gamma \quad i=1,2,3 \\
& \mathbf{D}_{\alpha \beta}^{0}\left(\mathbf{r}+\mathbf{l}_{i}\right)=\mathbf{D}_{\alpha \beta}^{0}(\mathbf{r}) \quad \alpha=\beta \text { and } \gamma \quad i=1,2,3
\end{aligned}
$$

Average:

$$
\left\langle\mathbf{D}_{\alpha \beta}^{0}\right\rangle^{\alpha}=-\varepsilon_{\alpha}^{-1} \mathbf{K}_{\alpha \beta}^{*} \quad \alpha=\beta \text { and } \gamma
$$

Problem A-II

$$
\begin{aligned}
& -\nabla \mathbf{d}_{\alpha \gamma}^{0}+\nabla^{2} \mathbf{D}_{\alpha \gamma}^{0}=\delta_{\alpha \gamma} \mathbf{I} \quad \text { in } V_{\alpha}, \quad \alpha=\beta \text { and } \gamma \\
& \nabla \cdot \mathbf{D}_{\alpha \gamma}^{0}=0 \quad \text { in } V_{\alpha}, \quad \alpha=\beta \text { and } \gamma \\
& \text { BC1 and BC2 } \quad \mathbf{D}_{\alpha \gamma}^{0}=0 \quad \text { at } A_{\alpha \sigma}, \quad \alpha=\beta \text { and } \gamma \\
& \text { BC3 } \quad \mathbf{D}_{\beta \gamma}^{0}=\mathbf{D}_{\gamma \gamma}^{0} \quad \text { at } A_{\beta \gamma} \\
& \text { BC4 } \quad \mu_{\beta} \mathbf{n}_{\beta \gamma} \cdot\left[-\mathbf{I d}_{\beta \gamma}^{0}+\left(\nabla \mathbf{D}_{\beta \gamma}^{0}+\nabla \mathbf{D}_{\beta \gamma}^{0 T}\right)\right] \\
& =\mu_{\gamma} \mathbf{n}_{\beta \gamma} \cdot\left[-\mathbf{I} \mathbf{d}_{\gamma \gamma}^{0}+\left(\nabla \mathbf{D}_{\gamma \gamma}^{0}+\nabla \mathbf{D}_{\gamma \gamma}^{0 T}\right)\right] \text { at } A_{\beta \gamma}
\end{aligned}
$$

Periodicity:

$$
\begin{aligned}
\mathbf{d}_{\alpha \gamma}^{0}\left(\mathbf{r}+\mathbf{l}_{i}\right) & =\mathbf{d}_{\alpha \gamma}^{0}(r) & \alpha=\beta \text { and } \gamma & i=1,2,3 \\
\mathbf{D}_{\alpha \gamma}^{0}\left(\mathbf{r}+\mathbf{l}_{i}\right) & =\mathbf{D}_{\alpha \gamma}^{0}(r) & \alpha=\beta \text { and } \gamma & i=1,2,3
\end{aligned}
$$

Average:

$$
\left\langle\mathbf{D}_{\alpha \gamma}^{0}\right\rangle^{\alpha}=-\varepsilon_{\alpha}^{-1} \mathbf{K}_{\alpha \gamma}^{*} \quad \alpha=\beta \text { and } \gamma
$$

Each of these two problems has basically a structure identical to that of a Stokes two-phase flow without any dependence on the curvature of the fluid-fluid interface. When a solver is available to compute the initial microscopic physical flow problem and locate the interface, the same solver in which inertial terms are removed can be used to solve problems A-I and A-II and determine the four tensors $\mathbf{K}_{\alpha \alpha}^{*}$ and $\mathbf{K}_{\alpha \kappa}^{*}(\alpha, \kappa=\beta, \gamma, \alpha \neq \kappa)$. When these four tensors are computed, the permeability and viscous drag tensors $\mathbf{K}_{\alpha}$ and $\mathbf{K}_{\alpha \kappa}(\alpha, \kappa=\beta, \gamma$, $\alpha \neq \kappa)$ can be determined according to

$$
\mathbf{K}_{\alpha}=\mathbf{K}_{\alpha \alpha}^{*}-\mathbf{K}_{\alpha \kappa}^{*} \cdot \mathbf{K}_{\kappa \kappa}^{*^{-1}} \cdot \mathbf{K}_{\kappa \alpha}^{*} \quad \alpha, \kappa=\beta, \gamma \quad \alpha \neq \kappa
$$




$$
\begin{aligned}
\mathbf{K}_{\alpha \kappa}= & \mathbf{K}_{\alpha \kappa}^{*} \cdot\left(\mathbf{I}-\mathbf{K}_{\kappa \kappa}^{*^{-1}} \cdot \mathbf{K}_{\kappa \alpha}^{*} \cdot \mathbf{K}_{\alpha \alpha}^{*^{-1}} \cdot \mathbf{K}_{\alpha \kappa}^{*}\right) \\
& \cdot\left(\mathbf{K}_{\kappa \kappa}^{*}-\mathbf{K}_{\kappa \alpha}^{*} \cdot \mathbf{K}_{\alpha \alpha}^{*^{-1}} \cdot \mathbf{K}_{\alpha \kappa}^{*}\right)^{-1} \quad \alpha, \kappa=\beta, \gamma \quad \alpha \neq \kappa
\end{aligned}
$$

\section{References}

Ahmed, N., Sunada, D.K.: Nonlinear flow in porous media. J. Hydr. Div. ASCE 95(HY6), 1847-1857 (1969)

Amaral Souto, H., Moyne, C.: Dispersion in two-dimensional periodic porous media, Part I: hydrodynamics. Phys. Fluids 9(8), 2243-2252 (1997)

Auriault, J.L.: Nonsaturated deformable porous media: quasistatics. Transp. Porous Media 2(1), 45-64 (1987)

Bear, J.: Dynamics of Fluids in Porous Media. Dover, New York (1972)

Beavers, G.S., Sparrow, E.M.: Non-Darcy flow through fibrous porous media. J. Appl. Mech. Transp. ASME 36, 711-714 (1969)

Bennethum, L., Giorgi, T.: Generalized Forchheimer equation for two-phase flow based on hybrid mixture theory. Transp. Porous Media 26(3), 261-275 (1997)

Blick, E.F.: Capillary orifice model for high speed flow through porous media. I and EC. Process Design Dev. 5, 90-94 (1966)

Buchlin, J., Stubos, A.: Phase change phenomena at liquid saturated self heated particulate beds. Application of Transport in Porous media, Von Karman Institute for Fluid Dynamics, vol. 4, pp. 221-276. RhodeSainte-Genèse, Belgium (1987)

Carbonell, R.G., Whitaker, S.: Heat and mass transfer in porous media. In: Bear, J., Corapcigolu, M.Y. (eds.) Fundamentals of Transport Phenomena in Porous Media, pp. 121-198. Martinus Nijhoff Publishers, Dordrecht (1984)

Chen, Z., Lyons, S.L., Qin, G.: Derivation of the Forchheimer law via homogenization. Transp. Porous Media 44(2), 325-335 (2001)

Coulaud, O., Morel, P., Caltagirone, J.P.: Numerical modeling of non-linear effects in laminar flow through a porous medium. J. Fluid Mech. 190, 393-407 (1988)

Cvetkovic, V.D.: A continuum approach to high velocity flow in a porous medium. Transp. Porous Media 1(1), 63-97 (1986)

Darcy, H.: Les fontaines publiques de la ville de Dijon. Librairie des corps impériaux des ponts et chaussées et des mines, Paris (1856)

Dullien, A.L., Azzam, M.I.S.: Flow rate-pressure gradient measurement in periodically nonuniform capillary tube. AIChE J. 19, 222-229 (1973)

Ergun, S.: Fluid flow through packed columns. Chem. Eng. Prog. 48, 89-94 (1952)

Evans, E.V., Evans, R.D.: Influence of an immobile or mobile saturation on non-Darcy compressible flow of real gases in propped fractures. J. Petrol. Technol. 40(10), 1343-1351 (1988)

Evans, R.D., Hudson, C.S., Greenlee, J.E.: The effect of an immobile liquid saturation on the non-Darcy flow coefficient in porous media. J. SPE Prod. Eng. Trans. AIME 283, 331-338 (1987)

Firdaouss, M., Guermond, J.L.: Sur l'homogénéisation des équations de Navier-Stokes à faible nombre de Reynolds. C. R. Acad. Sci. Paris, Série I 320, 245-251 (1995)

Firdaouss, M., Guermond, J.L., Le Quéré, P.: Nonlinear corrections to Darcy's law at low Reynolds numbers. J. Fluid Mech. 343, 331-350 (1997)

Firoozabadi, A., Katz, D.L.: An analysis of high-velocity gas flow through porous media. J. Petrol. Technol. February 1979, 211-216 (1979)

Firoozabadi, A., Thomas, L.K., Todd, B.: High-velocity flow in porous media. SPE Reserv. Eng. 10, 149-152 (1995)

Forchheimer, P.: Wasserbewgung durch boden. Z. Ver. Deutsch. Ing. 45, 1782-1788 (1901)

Fourar, M., Lenormand, R.: Inertial effects in two-phase flow through fractures. Oil Gas Sci. Technol. - Rev. IFP 55(3), 259-268 (2000)

Geertsma, J.: Estimating the coefficient of inertial resistance in fluid flow through porous media. Soc. Petrol. Eng. J. 14, 445-450 (1974)

Giorgi, T.: Derivation of the Forchheimer law via matched asymptotic expansions. Transp. Porous Media 29(2), 191-206 (1997)

Gray, W.G.: A derivation of the equations for multi-phase transport. Chem. Eng. Sci. 30, 229-233 (1975)

Hassanizadeh, S.M., Gray, W.G.: High velocity flow in porous media. Transp. Porous Media 2, 521-531 (1987)

Howes, F., Whitaker, S.: The spatial averaging theorem revisited. Chem. Eng. Sci. 40, 1387-1392 (1985) 
Irmay, S.: On the theoretical derivation of Darcy and Forchheimer formulas. J. Geophys. Res. 39, $702-707$ (1958)

Kalaydjian, F.: Origin and quantification of coupling between relative permeabilities for two-phase flows in porous media. Transp. Porous Media 5(3), 215-229 (1990)

Koch, J.B., Ladd, A.J.C.: Moderate Reynolds number flows through periodic and random arrays of aligned cylinders. J. Fluid Mech. 349, 31-66 (1997)

Lasseux, D., Whitaker, S., Quintard, M.: Determination of permeability tensors for two-phase flow in homogeneous porous media: theory. Transp. Porous Media 24(1), 107-137 (1996)

Lipinski, R.J.: A particle bed dryout model with upwind and downward boiling. Trans. Am. Nucl. Soc. 35, 350358 (1980)

Lipinski, T.J.: A model for boiling and dryout in particle beds. Report SAND 82-0756 (NUREG/CR-2646), Sandia Labs (1982)

Liu, X., Civan, F., Evans, R.D.: Correlations of the non-Darcy flow coefficient. J. Can. Petrol. Technol. 34(10), 50-54 (1995)

Lockhart, R., Martinelli, R.: Proposed correlation of data for isorthermal two-phase two-component flow in pipes. Chem. Eng. Prog. 45, 39 (1949)

Ma, H., Ruth, D.W.: The microscopic analysis of high Forchheimer number flow in porous media. Transp. Porous Media 13(2), 139-160 (1993)

MacDonald, I.F., El-Sayed, M.S., Mow, K., Dullien, F.A.L.: Flow through porous media, the Ergun equation revisited. Ind. Eng. Chem. Fund. 18(3), 199-208 (1979)

Marle, C.M.: Ecoulements monophasiques en milieu poreux. Rev. Inst. Frantais du Ptrole 22, 14711509 (1967)

Mei, C.C., Auriault, J.L.: The effect of weak inertia on flow through a porous medium. J. Fluid Mech. 222, 647-663 (1991)

Muskat, M.: The flow of homogeneous fluids through porous media. International Human Resources Development Corporation (reprint from McGraw-Hill, 1982), Boston (1937)

Papathanasiou, T.D., Markicevic, B., Dendy, E.D.: A computational evaluation of the Ergun and Forchheimer equations for fibrous porous media. Phys. Fluids 13(10), 2795-2804 (2001)

Quintard, M., Whitaker, S.: Transport in ordered and disordered porous media I: the cellular average and the use of weighting functions. Transp. Porous Media 14(2), 163-177 (1994a)

Quintard, M., Whitaker, S.: Transport in ordered and disordered porous media II: generalized volume averaging. Transp. Porous Media 14(2), 179-206 (1994b)

Quintard, M., Whitaker, S.: Transport in ordered and disordered porous media V: geometrical results for two-dimensional systems. Transp. Porous Media 15(2), 183-196 (1994c)

Raats, D.A.C., Klute, A.: Transport in soils: the balance of momentum. Soil Sci. Soc. Am. Proc. 32, 161166 (1968)

Rasoloarijaona, M., Auriault, J.L.: Nonlinear seepage flow through a rigid porous medium. Eur. J. Mech. B/Fluids 13(2), 177-195 (1994)

Rojas, S., Koplik, J.: Nonlinear flow in porous media. Phys. Rev. E 58(4), 4476-4782 (1998)

Rose, W.: Data interpretation problems to be expected in the study of coupled fluid flow in porous media. Transp. Porous Media 4(2), 185-189 (1989)

Ruth, D., Ma, H.: Numerical analysis of the viscous incompressible flow in a diverging-converging RUC. Transp. Porous Media 13(2), 161-177 (1993)

Scheidegger, A.E.: The Physics of Flow Through Porous Media. University of Toronto Press, Toronto, Canada (1974)

Skjetne, E., Auriault, J.L.: High-velocity laminar and turbulent flow in porous media. Transp. Porous Media 36, 131-147 (1999)

Skjetne, E., Hansen, A., Gudmundsson, J.S.: High velocity flow in a rough fracture. J. Fluid Mech. 383, 1-28 (1999)

Slattery, J.C.: Interfacial Transport Phenomena. Springer, New York (1990)

Thauvin, F., Mohanty, K.K.: Network modeling of non-Darcy flow through porous media. Transp. Porous Media 31(1), 19-37 (1998)

Torres, F.E.: Closure of the governing equations for immiscible two-phase flow: a research comment. Transp. Porous Media 2(4), 383-393 (1987)

Truesdell, C., Toupin, R.: The Classical Field Theories. Springer-Verlag, New-York (1960)

Ward, J.C.: Turbulent flow in porous media. J. Hydr. Div., ASCE 90(HY5), 1-12 (1964)

Whitaker, S.: Flow in porous media I: a theoretical derivation of Darcy's law. Transp. Porous Media 1(1), 3-25 (1986a)

Whitaker, S.: Flow in porous media II: the governing equation for immiscible two-phase flow. Transp. Porous Media 1(1), 105-125 (1986b) 
Whitaker, S.: The closure problem for two-phase flow in homogeneous porous media. Chem. Eng. Sci. 49, 765780 (1994)

Whitaker, S.: The Forchheimer equation: a theoretical development. Transp. Porous Media 25(1), 27-61 (1996) Whitaker, S.: The Method of Volume Averaging. Theory and Applications of Transport in Porous Media. Kluwer Academic, Dordrecht, The Netherlands (1999)

Wodie, J.C., Levy, T.: Correction non linéaire de la loi de Darcy. C. R. Acad. Sci. Paris II 157-161 (1991)

Zarcone, C.: Etude du couplage visqueux en milieu poreux: Mesure des perméabilités relatives croisées. Ph.D. Thesis, INP Toulouse (1994) 